\title{
Cyclin-dependent-like kinase 5 is required for pain signaling in human sensory neurons and mouse models
}

\author{
Paolo La Montanara ${ }^{1}$, Arnau Hervera ${ }^{1}$, Lucas Baltussen ${ }^{2}$, Thomas H Hutson ${ }^{1}$, Ilaria \\ Palmisano $^{1}$, Francesco De Virgiliis ${ }^{1}$, Guiping Kong ${ }^{1}$, Jessica Chadwick ${ }^{1}$, Yunan Gao ${ }^{3}$, \\ Katalin Bartus ${ }^{4}$, Qasim A. Majid ${ }^{5}$, Nikos Gorgoraptis ${ }^{1}$, Kingsley Wong ${ }^{6}$, Jenny Downs ${ }^{6}$, \\ Tommaso Pizzorusso ${ }^{7}$, Sila Ultanir ${ }^{3}$, Helen Leonard ${ }^{6}$, Hongwei $\mathrm{Yu}^{8}$, David S Millar ${ }^{9}$, Nagy \\ Istvan $^{10}$, Nicholas D Mazarakis ${ }^{3}$, Simone Di Giovanni $^{1,{ }^{*}}$ \\ ${ }^{1}$ Department of Brain Sciences, Division of Neuroscience, Imperial College London, London, UK \\ ${ }^{2}$ Kinases and Brain Development Laboratory, The Francis Crick Institute, London, UK \\ ${ }^{3}$ Gene Therapy, Centre for Neuroinflammation and Neurodegeneration, Division of Brain \\ Sciences, Department of Medicine, Imperial College London, London, UK \\ ${ }^{4}$ The Wolfson Centre for Age-Related Diseases, King's College London, Guy's Campus, London \\ Bridge, London, UK \\ ${ }^{5}$ National Heart and Lung Institute, Imperial College London, W12 0NN, UK \\ ${ }^{6}$ Telethon Kids Institute, The University of Western Australia, Perth, WA, Australia \\ ${ }^{7}$ Institute of Neuroscience, National Research Council (CNR), I-56124, Pisa, Italy and \\ Department of Neuroscience, Psychology, Drug Research and Child Health NEUROFARBA, \\ University of Florence, I-50135, Florence, Italy \\ ${ }^{8}$ Department of Anesthesiology, Medical College of Wisconsin WI 53226, Milwaukee, USA \\ 9Institute of Cancer \& Genetics, Cardiff University, UK \\ ${ }^{10}$ Nociception, Section of Anesthetics, Pain Medicine and Intensive Care, Department of Surgery \\ and Cancer, Imperial College London, London, UK
}

\section{Abstract}

Cyclin-dependent-like kinase 5 (Cdk15) gene mutations lead to an X-linked disorder that is characterized by infantile epileptic encephalopathy, developmental delay and hypotonia. However, we found that a substantial percentage of these patients also report a previously unrecognised anamnestic deficiency in pain perception. Consistent with a role in nociception, we discovered that Cdk15 is expressed selectively in nociceptive dorsal root ganglia (DRG) neurons in mice and in iPS-derived human nociceptors. CDKL5 deficient mice display defective epidermal innervation and conditional deletion of Cdkl5 in DRG sensory neurons impairs nociception, phenocopying CDKL5 deficiency disorder in patients. Mechanistically, Cdk15 interacts with CaMKIIa to control outgrowth as well as TRPV1-dependent signaling, which are disrupted in both Cdk15 mutant

Correspondence to: Paolo La Montanara.

*Correspondence to: s.di-giovanni@imperial.ac.uk or p.la-montanara@imperial.ac.uk. 
murine DRG and human iPS-derived nociceptors. Together, these findings unveil a previously unrecognized role for Cdkl5 in nociception, proposing an original regulatory mechanism for pain perception with implications for future therapeutics in CDKL5 deficiency disorder.

\section{Introduction}

Mutations in the X-linked Cdkl5 gene, encoding the cyclin-dependent-like kinase 5, are associated with the CDKL5 Deficiency Disorder (CDD) that is characterized by early-onset intractable seizures, severe intellectual disability, and motor impairment $(1,2)$. Cdkl5 is strongly expressed in the central nervous system (CNS), particularly in neurons of the cortex and of the hippocampus (3). Defective Cdk15 impairs proper brain development, learning and memory, neuronal activity, including molecular processes related to neuronal depolarization and synapse formation (4-7). Cdk15 is activated by neuronal activity and it has also been implicated both in the phosphorylation and interaction with nuclear as well as cytoplasmic proteins potentially modulating gene expression and cytoskeleton changes ultimately affecting neuronal activity and cell survival $(2,5,8,9)$. Recently microtubule and centrosome-associated proteins have been identified as targets of Cdk15 kinase activity, including MAP1S, EB2, ARHGEF2, CEP131 and DLG5, whose regulation is likely to affect axonal transport, outgrowth and synaptic plasticity $(10,11)$.

CDD remains a disease without a cure, whose fundamental molecular and cellular mechanisms are still in need of much investigation and whose full clinical spectrum remains only partially characterized. An important current limitation is that unlike patients, CDD mutant mice do not develop epilepsy $(4,12-14)$. This makes the identification of Cdkl5dependent mechanisms or potential treatments with an impact on the human phenotype very challenging. Here, we discovered that CDD patients and animal models display an impairment in nociception and that Cdkl5 is required for peripheral nociceptive signaling in dorsal root ganglia (DRG) neurons in mice and in induced pluripotent cell (iPS)-derived human nociceptors. Mechanistically, conditional deletion of Cdkl5 in DRG sensory neurons impairs nociception, phenocopying CDKL5 deficiency disorder in patients. Lastly, we discovered that Cdkl5 interacts with CaMKIIa to control outgrowth of sensory neurons as well as TRPV1-dependent signaling including in iPS-derived neurons from CDD patients. Together, these findings reveal a previously unrecognized role for Cdk15 in nociception and they suggest an original peripheral regulatory mechanism for pain processing with implications for future therapeutic interventions in CDD.

\section{Results}

\section{Alteration in pain perception in CDD patients}

In a recent effort to broadly characterise the clinical phenotype, variation and natural history of CDD, the International CDKL5 Database (ICDD) (https://www.cdk15.com/cdk15international-registry-database/) was established in 2012. Included in the database are responses to questions given to caregivers about whether their child experiences alterations in pain perception. Analysis of this clinical data source allowed the identification of a previously unrecognized occurrence of alterations in pain perception. 
Patients in the ICDD were classified by age group, gender and mutation type (Table 1). Classification of individual CDKL5 mutations was based on predicted structural and functional phenotypic consequences similar to the groupings used in a previous study (15). In our analysis we have grouped together mutations leading to lack of functional protein and missense/in-frame mutations within catalytic domain and TEYmotif and compared them with mutations affecting the regulatory domains of Cdkl5 i.e. truncations after aa172.

Multinomial logistic regression was used to estimate the risk of altered pain sensitivity relative to no change in pain sensitivity (i.e. reduced pain sensitivity vs. normal pain sensitivity) in the overall sample, as well as in each category of age group, gender and mutation type (i.e. within category comparison) (Table 1). We then used the same model to evaluate the relative risk ratios of altered pain sensitivity in each category compared to the reference category (age group: 0-2 years, gender: female; mutation type: truncations after aa172) (i.e. between category comparison) (Table 1). Point estimates and their 95\% confidence interval were reported.

We found that altered pain sensitivity in their children was reported by $53.0 \%(122 / 230)$ of caregivers. Amongst these a total of 57.4\% (70/122) specifically reported decreased pain sensitivity, $19.7 \%$ (24/122) specifically reported enhanced sensitivity and 22.9\% (28/122) reported both. 202 caregivers provided specific responses including either reduced (70/202, $34.7 \%)$ or enhanced pain perception $(24 / 202,11.9 \%)$ (Table 1). Amongst those who reported either reduced or enhanced pain sensitivity, a total of $74.4 \%$ (70/94) reported decreased and $25.5 \%$ (24/94) increased pain perception. The likelihood of the response being specifically reduced pain perception was measured as risk ratio (RR) of reduced or enhanced pain sensitivity versus normal pain perception, whilst the relative risk ratio (RRR) was used to represent the relative change in the risk ratio in each category compared to the reference. As shown in Table 1 reduced pain perception was more likely to be reported than enhanced pain perception (RR 2.92, 95\% CI 2.89,7.00; $\mathrm{p}<0.001$ ). Compared with those aged two years or under, individuals aged six years and over were reported to have an increased likelihood of reduced sensitivity to pain (RRR 2.74, 95\% CI 1.36,5.53; $\mathrm{p}=0.005$ ). Moreover, compared with those with mutations in the Cdkl5 regulatory domain (truncations after aa172), those with a non-functional protein (including missense/in-frame mutations within catalytic domain and TEY motif) were also reported to have an increased likelihood of reduced sensitivity to pain (RRR $1.63,95 \%$ CI $0.87,3.06 ; \mathrm{p}=0.124$ ). However, we did not identify any differences by gender (RRR $1.03,95 \%$ CI $0.44,2.45 ; \mathrm{p}=0.941$ ). Reduced was significantly more likely than enhanced pain perception at each age group $(0-2$ years old, (RR 3.00, 95\% CI 1.28,7.06; $\mathrm{p}=0.012$ ); $3-5$ years old, (RR 2.67, 95\% CI 1.04,6.81; $\mathrm{p}=0.040) ; 6+$ years old, (RR 3.00, 95\% CI 1.52,5.94; $\mathrm{p}=0.002)$ and for each gene mutation group studied (no functional protein, (RR 2.94, 95\% CI 1.67,5.18; p<0.001); truncation after aa172, (RR 2.88, 95\% CI 1.29,6.42; $\mathrm{p}=0.010$ ), and for females (RR 3.16, 95\% CI 1.89,5.29; $\mathrm{p}<0.001)$.

\section{CdkI5 localizes to human and murine sensory neurons and is required for nociception}

Since primary nociceptors are localized in the dorsal root ganglia, we initially investigated whether Cdk15, so far localized to the CNS, was also expressed in the peripheral nervous 
system in murine as well as human nociceptors. Surprisingly, immuno and coimmunohistochemistry studies in DRG revealed that Cdk15 is indeed expressed and mainly found in the cytoplasm of small diameter DRG neurons (Fig. 1A-C), where the signal becomes detectable after the age of post-natal day P45 (fig. S1A,B). Around $90 \%$ of Cdkl5 positive neurons express the nociceptive markers CGRP or IB4, while only a small percentage express PARV or NF200 (Fig. 1D-F).

The expression of $C d k 15$ in murine DRG was confirmed by RT-PCR and immunoblotting (fig. S1C,D), where $C d k 15$ was expressed although to a lower degree compared to the brain. Additionally, Cdk15 mRNA neuronal expression was detected by RT-PCR from sorted GFP+ DRG neurons (fig. S1E,) in line with previous single cell studies (16) (17).

Next, in order to find whether Cdkl5 was also expressed in human nociceptors, we differentiated iPSC obtained from human skin biopsies into nociceptors. After having confirmed the differentiation of iPSC by immunolabelling for $\beta$ III-Tubulin as neuronal and CGRP as nociceptor marker respectively (fig. S2A-E), we observed that Cdk15 was indeed expressed in human nociceptors (Fig. 1G,H).

In order to directly address whether the expression of $C d k 15$ in DRG sensory neurons was required for nociception, we took advantage of the conditional deletion of Cdk15 in sciatic DRG neurons by injecting an AAV5-creGFP or control GFP virus to the sciatic nerve of Cdk15-floxed mice (12) (Fig. 2A, fig. S3A, B). Since the cre-virus reduces Cdk15 expression selectively in DRG neurons, the strong reduction of Cdk15 signal by immunoblotting strongly supports the selective neuronal expression in DRG ganglia as well as the efficiency of the deletion (Fig. 2B, C). Cdkl5 conditionally deleted mice showed an increase in paw withdrawal latency and threshold in response to noxious hot temperature (Haargraves plantar test) and noxious mechanical stimulation (Von Frey) respectively (Fig. 2D,F). We performed the same functional tests in Cdkl5 mutant mice, an established mouse model of CDD carrying a nonsense mutation (stop codon) leading to deletion of Cdk15-Ex6 (4). Here, we found a reduction in nociception compared to WT littermates that phenocopied what observed in Cdk15 conditionally deleted mice (Fig. 2E,G).

Importantly, in Cdk15 conditionally deleted mice additional sensory modalities were unaffected as indicated by the time-to-contact and the time-to-removal during the adhesive removal test (18) (Fig. 2H), and by the thermal place preference test, evaluating the nonnociceptive thermal sensitivity (fig. S3C). Sensorimotor functions as assessed by the gridwalk task (19) were also unaltered (Fig. 2I), suggesting a selective role for CDKL5 in nociception. Importantly, the number of nociceptors did not differ between WT and Cdk15 null mice (fig. S4).

Together, these data suggest that Cdk15 is required for physiological nociception that relies upon the expression of Cdk15 in DRG neurons. 


\section{Cdkl5 controls the outgrowth of human and murine sensory neurons via a CaMKII- dependent mechanism}

To investigate the signaling pathways involved in Cdk15-dependent pain transmission, we decided to identify interactors of Cdkl5 in vivo. To this end, we performed immunoprecipitation of Cdk15 from the murine cortex, where the kinase is highly enriched, followed by mass spectrometry of the eluate (Suppl. File 1 and fig. S5A). Considering the normalized protein ratios of each immunoprecipitation experiment $(n=6)$, we identified 23 proteins co-immunoprecipitating with Cdkl5 (fig. S5B). Interestingly, 74\% of them correspond to genes associated with neuronal activity, neural development and epilepsy (fig. S5C-F), supporting the physiological relevance of our pool of interactors. Top ranked Cdk15 co-immunoprecipitating proteins included CaMKIIa, putatively the strongest interactor of Cdk15, and some proteins associated with the neuronal cytoskeleton, such as Myh10 (20), Tubb3 (21) and Dynch1h1 (22-25) (fig. S5G). The association with CaMKIIa suggests also a role for Cdkl5 in calcium-dependent signaling, neuronal activity (26) and in cytoskeleton remodeling (27-32).

Previous immunohistochemical studies have found that CaMKII is expressed in DRG nociceptors (33), where it plays a role in the regulation of neurite outgrowth (34). In particular, CaMKII is expressed in TRPV1-immunoreactive nociceptors, in both CGRP+ and IB4+ subpopulations, where it is required for capsaicin-mediated nociception via modulation of TRPV1; capsaicin stimulation of TRPV1 and calcium entry activate CaMKII that is in turn required to potentiate TRPV1 signaling $(35,36)$. Hence, we hypothesised that Cdk15 could be a partner of CaMKIIa in the regulation of pain both via modulation of cytoskeleton remodelling and nociception signaling pathways.

First, we examined by confocal microscopy the co-expression of Cdk15, CaMKIIa and TRPV1 in DRG neurons and we found that they co-localize in most DRG neurons (Fig. 3AC, fig. S6). Next, we confirmed, by co-immunoprecipitation and immunoblotting from brain extracts, that Cdk15 does interact with CaMKIIa in vivo (fig. S5H). Similarly, coimmunoprecipitation experiments after overexpression of CDKL5 in HEK293 cells, or of its inactive kinase dead form CDKL5-K42R and CaMKIIa, confirmed the interaction between the active form of CDKL5 and CaMKIIa. Importantly, no interaction was observed between CaMKIIa and CDKL5-K42R (fig. S5I), which showed lack of kinase activity in an in vitro kinase assay (fig. S5J), supporting the specificity of the findings. We finally established the molecular proximity between Cdkl5 and CaMKIIa in DRG neurons by using the Proximity Ligation Assay (Fig. 3D,E), supporting the co-expression and co-immunoprecipitation data.

We next investigated whether Cdk15-CaMKIIa signaling axis would be required for the outgrowth of DRG neuronal processes. Assessment of neurite outgrowth was carried out in both murine and human iPSC derived nociceptors from Cdk15 null mice and CDKL5 deficiency disorder patients. We found that cultured DRG neurons from Cdk15 mutant mice display impaired neurite outgrowth in CGRP positive neurons (Fig. 4A,C), and this is rescued to the WT amounts by viral-mediated overexpression of CDKL5 (Fig. 4E; fig. S7). On the contrary, outgrowth in proprioceptive parvalbumin positive neurons remained unaffected by Cdkl5 loss of function (Fig. 4B,D), indicating a specific role for Cdkl5 in nociceptors. Importantly, impaired neurite outgrowth was also observed in iPSC-derived 
nociceptive DRG neurons (CGRP+) obtained from skin biopsies of CDKL5 patients, compared with the respective isogenic controls (Fig. 4F,G). CGRP+ neurons represent $30-40 \%$ of WT and mutant DRG neurons and more than $90 \%$ of iPSC derived sensory neurons were $\beta$ III-Tubulin+ (fig. S2D). Capsaicin responding cells were $\sim 60 \%$ of the $\mathrm{KCl}$ responding murine DRG cultured neurons, while they were more than $90 \%$ of the $\mathrm{KCl}$ responding iPSC derived neurons, both in mutant and isogenic cultures, indicating functionality of the nociceptors (fig. S2E). Interestingly, we found that neurite outgrowth was impaired in neurons derived from patients carrying a Cdk15 mutation compromising the kinase active site and the TEY motif, but not in neurons where only the terminal part of the Cdk15 tail is lost (Fig. 4H; Table 1).

Next, we asked whether CaMKII activity was required for Cdkl5-dependent neurite outgrowth. Indeed, we found that DRG outgrowth was significantly impaired following administration of the specific CaMKII enzymatic inhibitor KN93, while its inert structural analog KN92 did not have an effect (Fig. 5A,B). Interestingly, KN93 did not further reduce outgrowth in Cdk15 mutant cells (Fig. 5A,B), indicating that CDKL5 and CaMKII belong to the same signaling pathway. Importantly, CDKL5 overexpression rescued outgrowth defects in Cdk15 mutant knockout DRG neurons, but this effect was blocked by KN93. These data together suggest that Cdk15-dependent DRG outgrowth relies on CaMKII.

Since epidermal innervation is needed for proper nociception and it is a dynamic process requiring continuous cytoskeleton remodeling and outgrowth, we examined if Cdkl5 deficiency was associated with a defect in epidermal innervation.

Indeed, immunohistochemical analysis of skin innervation revealed impairment in epidermal but not dermal innervation in Cdk15 mutant versus WT adult mice (Fig. 6A-E). We also measured epidermal innervation from the post-natal day 4 (P4) to adulthood (P70) and found that $C d k 15$ mutant mice display deficient epidermal innervation only in adulthood (P70), and not at earlier stages (P4 or P16; Fig. 6B). In support of these data, we found, by immunoblotting of skin lysates, that the specific neuronal and axonal proteins PGP9.5, CGRP and $\beta$ III-tubulin are reduced in Cdk15 mutants as compared to WT adult mice (Fig. $6 \mathrm{~F}, \mathrm{G})$. However, consistent with the selective reduction of nociceptive fibres in the epidermis of Cdkl5 mutant mice, the expression of these axonal proteins is not reduced in sciatic nerve lysates (Fig. 6H,I) or after immunostaining for CGRP (Fig. 6J,K), in Cdk15 mutant mice.

\section{CdkI5 is required for TRPV1/CaMKII-dependent signaling and nociception in sensory neurons and in vivo}

Since TRPV1 activity relies on CaMKII-dependent interaction and phosphorylation $(35,36)$, we hypothesized that TRPV1 signaling might require Cdk15. Therefore we aimed to address whether Cdk15 and CaMKII regulate TRPV1-dependent calcium signaling after stimulation with the well-established ligand capsaicin, which activates nociception by engaging with its receptor TRPV1 (37-39). We used capsaicin as specific activator of TRPV1 and we measured calcium influx in iPS derived nociceptors to find that capsaicin-induced increase in calcium is impaired in neurons carrying CDKL5-mutations involving the kinase active domain (E55fsX74) and the TEY motif (G155fsX197) (Fig. 5C). Similarly, capsaicininduced calcium responses were impaired in Cdk15 mutant murine DRG explants and 
cultured neurons, where we established that overexpression of AAV9-CDKL5 rescued the defect in calcium response (Fig. 5D,E,H). When we blocked CaMKII signaling in cultured WT DRG neurons by using KN93, in contrast to its inert structural analog KN92, we observed, as expected, that KN93 inhibited the activation of TRPV1 after administration of capsaicin. Inhibition was similar to what observed in $C d k 15$ mutant cells, where the CaMKII inhibitor did not further decrease calcium amount (Fig. 5E). Delivery of KN93 after overexpression of CDKL5 in Cdk15 mutant DRG neurons blocked the rescue in calcium mobilization after capsaicin delivery (Fig. 5F). Together, these data confirm our initial hypothesis that CaMKII is required for Cdkl5-dependent calcium responses after capsaicin.

To further test the role of CaMKII signaling in Cdkl5 function, we infected cultured WT and Cdk15 mutant DRG neurons with AAV particles expressing the CaMKII-AIP (Autocamtide-2-related-Inhibitor Peptide) plasmid (40), or an active CDKL5 plasmid to rescue Cdk15 function in null neurons. We then measured neurite outgrowth and calcium following capsaicin stimulation (fig. S8).

CaMKII-AIP infected neurons showed impaired neurite outgrowth (fig. S8A,B) and reduced capsaicin-dependent calcium mobilization (fig. S8C,D) to a similar degree as Cdk15 mutant knockout DRG neurons. Both outgrowth and calcium were not further impaired in Cdk15 mutant knockout DRG neurons after CaMKII-AIP infection, suggesting functional interaction of the two proteins. Lastly and importantly, neurite outgrowth and capsaicindependent calcium induction were rescued by overexpression of Cdk15 (fig. S8A-D); however, this failed when CaMKII was inhibited (fig. S8A-D), indicating that CaMKII is required to mediate Cdkl5 activity.

Finally, we hypothesized that capsaicin signaling would also be compromised in vivo leading to biochemical and behavioral impairments in $C d k 15$ deficient mice. In line with this hypothesis, we found that both the licking behavior and cell signaling response to intradermal injection of capsaicin were impaired in vivo in $C d k 15^{-1 y}$ mutant mice and following conditional deletion of Cdk15 specifically in sciatic DRG sensory neurons. In fact, the time spent licking the right paw after injection of capsaicin $(1 \mu \mathrm{g})$ was significantly reduced both in $C d k 15^{-1 y}$ mice and in AAV5-CRE vs AAV5-GFP treated $C d k 15$-floxed mice (vehicle was injected in the left paw) (Fig. 7A-C). Consistently, five minutes after capsaicin injection in the right paw, pCaMKIIa was upregulated in DRG on the injected side. in WT but not in $C d k 15^{-/ y}$ mice, supporting a defective signaling response (Fig. 7D-F). We confirmed defective transmission of the stimulus in $C d k 15^{-/ y}$ mice also to the right dorsal horn of the spinal cord by measuring pERK $1 / 2$ expression, typically activated by capsaicinTRPV1 signaling in lamina I-II of the dorsal horn (41). Indeed, pERK1/2 was induced only in WT but not in $C d k 15^{-/ y}$ mice following intradermal injection of capsaicin (Fig. 7G-I) (42, 43).

\section{Discussion}

Our data reveal a previously unknown function of Cdk15 in the regulation of primary nociception in the peripheral nervous system as shown by impaired nociception following conditional deletion of Cdk15 in DRG sensory neurons. Specifically, we found that Cdk15 is 
required for CaMKII-dependent TRPV1 signaling and outgrowth of sensory neurons in both CDD murine and human neurons. In vivo, in a CDD animal model, this translates into impaired capsaicin-dependent nociceptive signaling and behavioral responses as well as in reduced epidermal innervation.

The anatomical, mechanistic and functional data all together support the initial anamnestic evidence that over $34 \%$ of CDKL5 patients have defective pain perception. The clinical questionnaire we employed should not be considered as an exhaustive clinical piece of data per se but rather a clinical indication that generated a hypothesis guiding further experiments in animal models and human iPSC. In a much smaller percentage of patients with enhanced pain perception, cortical hyperexcitability, which is a typical feature of these patients, might lead to dysfunctional central processing of nociception. This might be in line with disorders affecting cognition and neuronal activity in the central nervous system such as ASD and Rett syndrome (RTT), where it has been proposed that altered pain sensitivity is related to a dysfunctional mode of cortical pain processing, rather than to defective primary peripheral nociception (44) (45-48). Therefore, it is possible that modest impairment in peripheral pain perception and marked cortical hyperexcitability might coexist in a subset of patients leading to the net effect of enhanced pain perception. Alternatively and similarly to the protein SHANK3 that regulates TRPV1 and heat pain as a loss-of-function(49) and touch sensation as a gain-of-function in $\mathrm{ASD}(50)$, we cannot rule out that Cdk15 might have a dual loss and gain-of-function role in specific sensory neuron subtypes.

CDD and RTT are distinct diseases caused by mutations in separate independent genes, Cdk15 and methyl $\mathrm{CpG}$ binding protein $2(\mathrm{MeCP} 2)$ respectively. While in vitro data suggested that the two might interact $(1,51,52)$, functional interaction in vivo remains unclear.

Recently, genes such as $M e C P 2$ and CaMKIIa, which have been implicated in the pathogenesis of ASD (53-55), and that are highly expressed in the CNS, have also been localized to the peripheral nervous system where they regulate sensory modalities $(40,56)$. However, a role in nociception had not been described so far.

Limitations of our work include the need for future comprehensive clinical and neurophysiological investigation to fully characterize pain perception in CDD patients as well as for the assessment of potential additional Cdk15-dependent mechanisms that might control pain perception and nociception. Additionally, it is presently not known whether Cdk15 plays a role in inflammatory or in traumatic painful conditions or whether reduced epidermal innervation is directly responsible for impaired nociception. Lastly, while impairment in epidermal innervation only occurred in adult mice, the human clinical data includes infants and toddlers. Published evidence suggests that skin innervation is already complete approximately at 1.5 years of age (57), however it would be useful to generate data allowing for a direct comparison between the maturation of skin innervation in humans and mice.

In summary, the importance of these findings lies on the (i) identification of a previously unrecognized regulatory mechanism for nociception that relies on Cdk15, the (ii) discovery 
of impaired nociception as a previously uncharacterized symptom in CDD and (iii) the usefulness of nociception as therapeutic outcome measure in animal models of CDD. Since animal models of CDKL5 deficiency do not develop seizures, monitoring pain responses during therapeutic interventions might be a unique opportunity to test novel diseasemodifying treatment in pre-clinical before clinical settings. Lastly, our data suggest that gene therapy or other interventions in CDKL5 patients should not only be directed to the brain but also to dorsal root ganglia to restore nociceptive input in patients with impaired pain perception.

\section{Research Design and Methods}

\section{Study design}

We investigated the role of Cdkl5 in nociception in both human and murine sensory neurons as well as in Cdkl5 mutant mice by performing both mechanistic and behavioral studies.

\section{Questionnaire}

Ethics approval was obtained from the Human Research Ethics Committee, University of Western Australia.

\section{Mice}

All animal procedures were approved by Imperial College London ethic committee and were performed in accordance with the UK Animals Scientific Procedures Act (1986). C57B1/6 background mice lacking $C d k 15$ exon 6 (4) and Cdk15 floxed C57B1/6 background mice containing loxP sites flanking Cdk15 exon 4 (12) and wild-type littermates or C57Bl/6 (Harlan, UK) mice ranging from day-4 to day-70 of age were used for all experiments. Mice were anaesthetized with isoflurane (3\% induction, $2 \%$ maintenance) and buprenorphine (0.1 $\left.\mathrm{mg} \mathrm{kg}^{-1}\right)$ and carprofen $\left(5 \mathrm{mg} \mathrm{kg}^{-1}\right)$ were administered peri-operatively as analgesic. The experimenter was blind to the genotype of animals and to treatment given to each animal.

\section{Epidermal innervation}

Following euthanasia, the external skin of the mouse foot was sterilized with ethanol and a skin punch, $1 \mathrm{~mm}$ in diameter, was introduced perpendicularly to the surface of pad skin, rotated and advanced until reaching 1-2 mm depth (58). The specimens were immediately fixed in 3\% paraformaldehyde, $75 \mathrm{mM}$ Lysine hydrochloride (Sigma L5501), $2.2 \mathrm{mg} / \mathrm{ml}$ Sodium Metaperiodate (Sigma S1878) for up to 24 hours at $4{ }^{\circ} \mathrm{C}$, and then kept in a cryoprotective solution overnight. After fixation, the tissue was serially cut (sections of 50 $\mu \mathrm{m}$ in thickness, perpendicular to the dermis) using a freezing microtome. The slices were then treated with blocking solution for two hours at RT (PBS - Triton X-100 0.3\% - Donkey Serum 10\%) and exposed to different primary Abs. $\beta$ III-Tubulin (Abcam ab-18207) and Collagen IV (Sigma C1926) were the markers used to assess, respectively, the density of IENF and the basal lamina. Using confocal microscopy (Leica TCS SP5 II or Zeiss LSM-780 inverted confocal microscope), individual IENF crossing the dermal-epidermal junction were counted and the intraepidermal network length was analyzed by using Neuron-J software (magnification X20) in at least three non-consecutive sections (59). For anti-PGP9.5 immunohistochemistry, sections were immunostained by using Vectastain ABC 
kit (PK-4001) with minor adjustments. Endogenous peroxidase was blocked by incubation in PBS/0.1\% Tx 100 containing 0.3\% hydrogen peroxide for $10 \mathrm{~min}$. After 2x PBS washes, sections were blocked in normal blocking serum for $20 \mathrm{~min}$ and incubated with primary $\mathrm{Ab}$ (anti-PGP 9.5, Ultraclone, 1:20000, gift from Prof. Praveen Anand) overnight at R.T. After washing, sites of primary $\mathrm{Ab}$ binding were revealed by biotinylated secondary anti-rabbit incubation followed by avidin-biotin peroxidase reaction, using Impact DAB as substrate (SK-4105). Sections were counterstained for nuclei in Mayer's Haematoxylin, dehydrated with serial incubations of ethanol: 70\% (1x, 2min); $90 \%$ (1x, 2min); 100\% (2x, 5min), and mounted in xylene-based mounting medium.

\section{Murine DRG, Spinal Cord and Sciatic Nerve Immunohistochemistry}

After euthanasia, the tissues were removed and fixed in 4\%-PFA-PBS for up to $24 \mathrm{~h}$ at $4{ }^{\circ} \mathrm{C}$, and then kept in a cryoprotective solution overnight. After fixation, the samples were cut (thickness $15 \mu \mathrm{m}$ ) using a freezing microtome. Immunohistochemistry on tissue sections was performed according to standard procedures. For all antibodies used, the samples were blocked for $1 \mathrm{~h}$ with 10\% Donkey Serum - 0.3\% PBS-Triton-X100, and then incubated with Cdk15 (rabbit, Sigma HPA002847, 1:100), CGRP (mouse, abcam ab81887, 1:100), IB4 (Thermo-Fisher I21411 Alexa Fluor ${ }^{\mathrm{TM}} 488$ Conjugate, 1:250), Parvalbumin (mouse, abcam ab64555, 1:100), TRPV1 (guinea pig, Thermo-Fisher PA1-29770, 1:100), NF200 (mouse, N5389, Sigma Aldrich), CaMKIIa (mouse, Thermo-Fisher MA1-048, 1:100), phosphoThr286-CaMKIIa (mouse, Thermo-Fisher MA1-047, 1:100), phosphor-Thr202/Tyr204Erk1/2 (rabbit, Cell Signaling \#9101, 1:100); $\beta$ III-Tubulin (rabbit, abcam ab18207, 1:500) antibodies at $4{ }^{\circ} \mathrm{C}$ overnight. Subsequently, samples were incubated with AlexaFluorconjugated goat secondary antibodies according to standard protocols (Invitrogen). For triple immunostaining: $15 \mu \mathrm{m}$ slides incubated with primary antibodies CDKL5 anti rabbit 1:100, TRPV1 anti guinea pig 1:100, CamKIIa anti mouse 1:100 (secondary antibodies: Alexa 647 anti rabbit 1:1000, Alexa 594 anti mouse 1:1000, Alexa 488 anti guinea pig 1:100). All tissue sections were counterstained with Hoechst (Molecular Probes).

\section{Differentiation of iPSC Derived Nociceptors}

iPSC lines from four CDKL5 patients, carrying different mutations (E55fsX74, G155fsX197, Q347X, S855X; special gift from Prof. N. Mazarakis, Imperial College London, and Dr. D. Millar, Cardiff University), were cultured in Matrigel coated wells (Corning Membrane Matrix 354234) and mTeSR ${ }^{\mathrm{TM}} 1$ medium (STEMCELL Technologies)

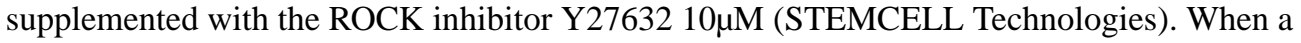
confluency of $60-70 \%$ was reached, we started the differentiation by using five inhibitors for

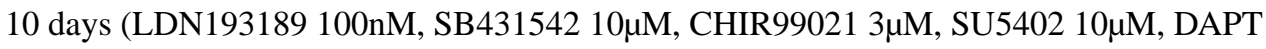
$10 \mu \mathrm{M}$; STEMCELL Technologies) in hESC medium (DMEM-F12, knockout serum replacement 20\% (Gibco), L-Glut 1mM, MEM non-essential amino acid solution (M7145 Sigma) (60). The cells were then grown in Neuronal Growth Medium (DMEM-F12, HI-FBS $10 \%$ ) supplemented with neurotrophins (BDNF 10ng/ml, NGF 10ng/ml, NT3 10ng/ml, GDNF 10ng/ml; STEMCELL Technologies) and ascorbic acid (35ng/ml; 72132 STEMCELL Technologies) for 4 days and treated with mitomycin C $(1 \mu \mathrm{g} / \mathrm{ml} ; 73272$ STEMCELL Technologies) for 3 hours. Lastly, the cells were grown for 30 days and replated 1 day before the calcium imaging experiment. The expression of neuronal markers 
( $\beta$ III-Tubulin and CGRP for nociceptors) was monitored at days 0, 7 and 12, as well as at day 42 , when we confirmed their sensitivity to $50 \mathrm{mM} \mathrm{KCl}$ and $500 \mathrm{ng} / \mathrm{ml}$ capsaicin. Cells were fixed with $4 \%$ PFA - $4 \%$ sucrose and the immunocytochemistry was performed by incubating fixed cells with $\beta$ III-tubulin (Abcam ab18207) and CGRP (Abcam ab81887) antibodies at $4{ }^{\circ} \mathrm{C}$ overnight. This was followed by incubation with AlexaFluor-conjugated goat secondary antibodies according to standard protocols (Invitrogen). All cells were counterstained with Hoechst (Molecular Probes).

\section{Western Blot analysis of skin, brain, DRG and sciatic nerve lysates}

Approximately 10-30 $\mu \mathrm{g}$ of protein extracts (lysis buffer: $50 \mathrm{mM}$ Tris- $\mathrm{HCl}, \mathrm{pH} 7.4,150 \mathrm{mM}$ $\mathrm{NaCl}$, Nonidet P-40 1\%, 1 mM EDTA, 0.5 mM DTT, protein inhibitor cocktail Sigma, 1 $\mathrm{mM}$ PMSF; lysed for $30 \mathrm{~min}$ on ice followed by 15 min centrifugation at $4^{\circ} \mathrm{C}$ ) from brain, DRG, sciatic nerve and skin were heated at $95^{\circ} \mathrm{C}$ and separated by $8 \%$ SDS-polyacrylamide gel electrophoresis (PAGE) gels (37.5:1 acrylamide:bis-acrylamide) and transferred to nitrocellulose membranes for $2 \mathrm{~h}$. Before gel loading, protein concentration was quantified using Pierce BCA Protein Assay Kit (ThermoScientific). Membranes were blocked with 5\% BSA or milk for $1 \mathrm{~h}$ at room temperature and incubated with Cdkl5 (Sigma HPA002847, 1:1000), CaMKIIa (AvivaSysBio Ab286 OAECOO614, 1:1000), CGRP (Abcam ab-189786, 1:1000), PGP9.5 (Abcam ab-8189, 1:1000), ßIII-tubulin (Abcam ab-18207, 1:1000), Gapdh (Cell Signaling \#2118) antibodies at $4^{\circ} \mathrm{C}$ overnight. Following HRP-linked secondary antibody (GE Healthcare) incubation for $1 \mathrm{~h}$ at room temperature, membranes were developed with ECL substrate (GE Healthcare) (ThermoScientific) and the bands detected with a CCD camera (Syngene, GeneGnome XRQ) and analyzed, after subtraction of the background, by using the Image J software.

\section{RNA isolation and RT-PCR Analysis}

Total RNA was isolated using the RNeasy Mini Kit (Qiagen) according to the manufacturer's instructions. Contaminating DNA was removed with DNaseI (Invitrogen 1734510), and the obtained RNA was quantified with a NanoDrop UV Visible Spectrophotometer; the quality of the RNA was assessed through agarose gel electrophoresis. cDNA was synthetized from 200 ng of RNA using the SuperScript First Strand reverse transcriptase kit (Invitrogen 1933910) as indicated by the manufacturer. The RT-PCR was performed in $20 \mu \mathrm{l}$ using $50 \mathrm{ng}$ of cDNA and the following cycling parameters: Cdk15-Ex 6 and Gapdh, 35 cycles, $95{ }^{\circ} \mathrm{C} 1 \mathrm{~m}$, $54{ }^{\circ} \mathrm{C} 30 \mathrm{~s}, 72{ }^{\circ} \mathrm{C} 1 \mathrm{~m}$.

\section{Injection of viral vectors into the sciatic nerve}

$2.5 \mu \mathrm{l}$ of each viral vector (AAV5-GFP SignaGen SL100819 or AAV5Cre SignaGen SL100821, titer $3.06 \times 10^{13} \mathrm{GC} / \mathrm{ml}$ ) was injected into the sciatic nerve of adult $C d k 15$-floxed mice (12) with a Hamilton syringe and Hamilton needle (NDL small RN ga34/15mm/ pst $45^{\circ}$ ). After 2 months, the animals were exposed to specific behavioral tests and then sacrificed, to evaluate the silencing of Cdk15gene in sciatic DRG by WB. 


\section{Mechanical Allodynia Test}

Mechanical allodynia was quantified by measuring the hind paw withdrawal response to von Frey filament stimulation. Animals were placed in methacrylate cylinders $(20 \mathrm{~cm}$ high, $9 \mathrm{~cm}$ diameter) with a wire grid bottom through which the von Frey filaments (North Coast Medical, Inc., San Jose, CA) with a bending force in the range of 0.16-8g were applied by using a modified version of the up-down paradigm, as previously reported(61). The filament of $0.16 \mathrm{~g}$ was used first and the 8-g filament was used as a cut-off. Then, the strength of the next filament was decreased or increased according to the response. The threshold of response was calculated using a regression curve generated based on the sequence of filament number (numbered from 1 to 9 ) versus $\log (10)$ of their strength in gr, an interpolation of the number of filament plus or minus a correction factor of 0.5 according to the response was then used to calculate the threshold of response in gr. Clear paw withdrawal, shaking, or licking of the paw was considered as a nociceptive-like response. Both hind paws were tested and averaged. Animals were allowed to habituate for $1 \mathrm{~h}$ before testing in order to allow an appropriate behavioral immobility.

\section{Hargreaves Test (Plantar Test)}

Thermal hyperalgesia was assessed as previously reported by Hargreaves et al. Paw withdrawal latency in response to radiant heat was measured using the plantar test apparatus (Ugo Basile, Varese, Italy). Briefly, the mice were placed in methacrylate cylinders $(20 \mathrm{~cm}$ high $\times 9 \mathrm{~cm}$ diameter) positioned on a glass surface. The heat source was positioned under the plantar surface of the hind paw and activated with a radiant light beam, the intensity of which was chosen in preliminary studies to give baseline latencies from 8 to $9 \mathrm{~s}$ in control mice. A cut-off time of $15 \mathrm{~s}$ was used to prevent tissue damage in the absence of response. The mean paw withdrawal latencies from both hind paws were determined from the average of five separate trials, taken at 2-min intervals to prevent thermal sensitization and behavioral disturbances. Animals were habituated to the environment for $1 \mathrm{~h}$ before the experiment to become quiet and to allow testing (62).

\section{Grid walk}

Mice were allowed to run the grid walk $(50 \times 5 \mathrm{~cm}$ plastic grid $(1 \times 1 \mathrm{~cm})$ placed between two vertical 40-cm-high wood blocks) three times per session. The total number of steps and missteps per run for each hindpaw was analyzed by a blinded investigator.

\section{Adhesive removal}

Small adhesive stimuli (6-mm round adhesive labels) were placed on both hindpaws of the mouse, the time to make first contact with both forepaws, as well as the time to completely remove the adhesive were recorded for each paw. Each mouse underwent three trials. All testing was performed by a blinded investigator, after a period of acclimatization.

\section{Thermal Place Preference Test}

Unrestrained mice were allowed to move freely between two connected plate compartments (Ugo Basile) each set at a given temperature, thus choosing their preferred temperature environment. Mice were placed on one plate set to $30^{\circ} \mathrm{C}$, which is known to be a 
thermoneutral temperature for mice while the other plate was set to either neutral $30^{\circ} \mathrm{C}$ or non-noxious temperatures of either 23 or $37^{\circ} \mathrm{C}$. Each animal was monitored for 10 minutes, recording the time spent in each plate compartment. Each temperature range was tested at the same time of day on each testing day, randomly interchanging plate compartments to avoid environmental bias. All testing was performed by a blinded investigator, after a period of acclimatization.

\section{Cdkl5 immunoprecipitation}

The cortex of three mice (P65) was removed and immediately lysed in lysis buffer (Tris-HCl $7.520 \mathrm{mM}, \mathrm{NaCl} 150 \mathrm{mM}$, EDTA 1mM, EGTA 1mM, Triton X-100 0.1\%, PIC Sigma P8340-1x, 1mM DTT, PhosSTOP Sigma P0044-1x) on ice (glass pestle Thermo-Fisher FB56679) and centrifuged (14000g 15m). The protein concentration was determined by the Bradford method. For each sample, $1 \mathrm{mg}$ of protein was treated for $30 \mathrm{~m}$ with $500 \mathrm{U}$ Benzonase (E1014 Sigma) at $37^{\circ} \mathrm{C}$, then exposed (overnight wheel, $4^{\circ} \mathrm{C}$ ) to $1 \mu \mathrm{g}$ of Cdk15Ab (Sigma HPA002847) or 1 $\mu$ g Rabbit IgG (Jackson 011000003 lot95014). Magnetic beads (40 $\mu 1$ 50\% Dynabeads Protein G 10003D, Life Technologies) were subsequently added to the samples, and the mixture was further incubated on the wheel for $2 \mathrm{hrs}$ at $4^{\circ} \mathrm{C}$. The beads were washed three times $\left(3 \times 20 \mathrm{~m}\right.$ washes with lysis buffer, wheel $\left.4^{\circ} \mathrm{C}\right)$ and proteins were

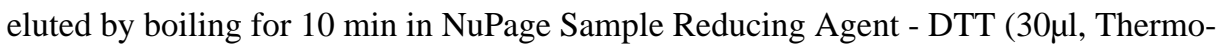
Fisher NP0009; $80^{\circ} \mathrm{C}$ ) and loaded on a $8 \%$ SDS-PAGE or analyzed by Mass Spectrometry.

\section{Mass Spectrometry Sample Preparation}

Samples were boiled at $70^{\circ} \mathrm{C}$ for 10 minutes in 1x NuPAGE LDS Sample Buffer (Life technologies) containing 100mM DTT and separated on a 10\% NuPAGE Bis-Tris gel (Life technologies) for 10 at $180 \mathrm{~V}$ in MES running buffer (Life technologies). After fixation in $7 \%$ acetic acid containing $40 \%$ methanol and subsequently staining for 30 minutes using Colloidal Blue staining kit (Life technologies) protein lane was excised from the gel, chopped and destained (50\% ethanol in $25 \mathrm{mM} \mathrm{NH4HCO3)} \mathrm{for} 15$ minutes rotating at room temperature and dehydrated for 10 minutes rotating in $100 \%$ acetonitrile. Vacuum dried samples were rehydrated and reduced for 60 minutes in reduction buffer $(10 \mathrm{mM}$ DTT in $50 \mathrm{mM} \mathrm{NH} 4 \mathrm{HCO} 3 \mathrm{pH} 8.0$ ) at $56^{\circ} \mathrm{C}$ and subsequently alkylated in $50 \mathrm{mM}$ iodoacetamide in $50 \mathrm{mM}$ NH4HCO3 $\mathrm{pH} 8.0$ for 45 minutes at room temperature in the dark. Dehydrated and vacuum dried samples were trypsin digested $(1 \mu \mathrm{g}$ trypsin/sample in $50 \mathrm{mM}$ Triethylammonium bicarbonate buffer $\mathrm{pH} 8.0$ ) at $37^{\circ} \mathrm{C}$ overnight. Stepwise peptide extraction was done as follows: twice extraction solution (30\% acetonitrile) and $100 \%$ acetonitrile for 15 minutes at $25^{\circ} \mathrm{C}$ shaking at $1400 \mathrm{rpm}$. Reductive methylation for quantification was performed as described in Hsu et al. (2003) (63). For Cdk15 immunoprecipitation, peptides were labelled LIGHT (DimethLys-0 and DimethNter0) and control IgG immunoprecipitation peptides labelled HEAVY (DimethLys-4 and DimethNter4). Afterwards they were mixed 1:1 and further processed. In the so called "reverse" experiment Cdk15 IP peptides were labelled HEAVY and control IgG IP peptides were labelled LIGHT. We performed Cdk15 IP experiments in duplicates for each of the three different animals resulting in $\mathrm{n}=6$. After purification and desalted using $\mathrm{C} 18$ stage tips (Empore) (64) $3.5 \mu \mathrm{L}$ peptides were loaded and separated on $\mathrm{C} 18$ column (New Objective) 
with $75 \mu \mathrm{m}$ inner diameter self-packed with $1.9 \mu \mathrm{m}$ Reprosil beads (Dr. Maisch) which was mounted to an EasyLC1000 HPLC (Thermo-Fisher Scientific).

\section{Mass Spectrometry Measurement and Data Analysis}

Reversed-phase chromatography gradient (Buffer A: 0.1\% formic acid, Buffer B: $80 \%$ acetonitrile and $0.1 \%$ formic acid, Gradient: 0-67 min 0-22\% Buffer B, 67-88 min 22-40\% Buffer B, 89-92 min 40-95\% Buffer B) was applied and peptides eluted and directly sprayed into a Q Exactive Plus mass spectrometer from Thermo-Fisher Scientific operating in positive scan mode with a full scan resolution of 70,000; AGC target $3 \times 10^{\wedge} 6$; max IT = 20ms; Scan range $300-1650 \mathrm{~m} / \mathrm{z}$ and a Top10 MSMS method. Normalized collision energy was set to 25 and MSMS scan mode operated with resolution of 17,000; AGC target 1x10^5; $\max$ IT $=120 \mathrm{~ms}$. Triggered MSMS masses were excluded dynamically for 20 seconds. Database search was performed using MaxQuant Version 1.5.2.8 (65) against Mus Musculus Uniprot database (downloaded 8th January 2018; 53819 entries) with Trypsin/P as digestion enzyme allowing 2 missed cleavages. As settings the following was applied: variable modification: Acetyl (Protein N-term); Oxidation (M), fixed modifications:

Carbamidomethyl (C), FDR of $1 \%$ on peptide and protein amount was applied. As light label: DimethylLys0 and DimethylNter0 and heavy label: DimethylLys4 and DimethylNter4 were set with max. 3 labeled amino acids. Proteins with at least two unique peptides were considered as identified. Proteins matching reverse database or common contamination list as well as proteins with peptides only identified by peptides with modification were filtered out. Statistical calculation and visual presentation were done in $\mathrm{R}$ version 3.5.1 (released $2^{\text {nd }}$ July 2018; R Core Team (2016) R: A language and environment for statistical computing R: Foundation for Statistical Computing, Vienna, Austria. URL https://www.R-project.org/). Protein ratios of each immunoprecipitation experiment $(n=6)$ were divided by median to normalized ratios. The new obtained median was 1 . All "reverse" replicates were inverted to obtain, positive values for IP enrichment. Log2 transformed median normalized ratios were plotted as box plot (function "ggplot", package "ggplot2"). For each individual protein depicted in the box plot a parametric Two-tailed tests was performed (function "compare_means", package "ggpubr") against all other measured proteins. Significance was calculated and indicated $(* P<0.05 * * P<0.01 * * * P<0.001 * * * * P<0.0001)$. Further packages used: "openxlsx", "dplyr", "reshape". These studies were performed in the proteomics facility in Mainz, Germany.

\section{DuoLink Proximal ligation Assay}

After blocking DRG slices (50 $\mu \mathrm{m}$ of thickness), from P65 C57B1/6 Cdk15 null mice and wild-type littermates with Duolink Blocking Solution, primary antibodies raised in two different species, specific for the two proteins of interest (rabbit-Cdk15 Sigma HPA002847 and mouse-CaMKIIa Thermo-Fisher MA1-048), were added to the tissue samples (1:100) and incubated in a humidity chamber overnight at $4^{\circ} \mathrm{C}$. After washing, according to the manufacturer protocol (PLA; Sigma DUO92101), the samples were then incubated with secondary antibodies coupled to oligonucleotides (proximity probes) for 1 hour at $37^{\circ} \mathrm{C}$ and exposed to the ligase for 30 minutes at $37^{\circ} \mathrm{C}$; the DNA polymerase was lastly added for 100 minutes at $37^{\circ} \mathrm{C}$. The slides were washed thereafter, mounted and analyzed at the Leica TCS SP5 II confocal microscope (63x magnification; 6 non-consecutive $38 \mu \mathrm{m}$-sections from the 
entire DRG). Each DRG was cut throughout and 6 slices (1 out of 3) was used for immunolabelling experiments; imaging was carried out with the Leica TCS SP5 II confocal microscope. The number of PLA+ neurons per $\mathrm{mm}^{3}$ was counted.

\section{DRG culture}

Adult DRG were dissected and collected in Hank's Balanced Salt Solution (HBSS) on ice and digested according to standard procedures. Cultures were maintained in medium containing B27 and penicillin-streptomycin in DMEM:F12. Cells were plated on coated glass coverslips ( $\left.0.01 \mathrm{mg} \mathrm{ml}^{-1} \mathrm{PDL}\right)$ for $48-72 \mathrm{~h}$ and fixed with $4 \%$ PFA-4\% sucrose. Immunocytochemistry was performed by incubating fixed cells with Cdk15 (Sigma HPA002847), $\beta$ III-tubulin (Abcam ab18207), CaMKIIa (Thermo-Fisher MA1-048), (Abcam ab81887), Parvalbumin (Abcam ab555), GFP (Thermo-Fisher A-6455), HA (Abcam ab18181) antibodies at $4{ }^{\circ} \mathrm{C}$ overnight. This was followed by incubation with AlexaFluor-conjugated goat secondary antibodies according to standard protocols (Invitrogen). All cells were counterstained with Hoechst (Molecular Probes).

\section{DRG explants}

DRG from adult WT and $C d k 15$ null mice were isolated, plated on a culture dish and incubated in $\mathrm{Ca}(2+)$ dye Fluo-4AM (Thermo-Fisher F14201 494/506nm; $1 \mu \mathrm{M}$ ) in HBSS (with 20mM Hepes pH7.4 and 1 $\mu \mathrm{l} / \mathrm{ml}$ pluronic F127 P3000MP, Life Technologies) for 30m at $37^{\circ} \mathrm{C}$. After washing in HBSS-Hepes, the DRG were placed on the surface of polystyreneplates, perfused with a small amount of "basal medium" $(2 \mathrm{ml} / \mathrm{m}: \mathrm{NaCl} 160 \mathrm{mM}, \mathrm{KCl}$ 2.5mM, $\mathrm{CaCl}_{2} 1 \mathrm{mM}, \mathrm{MgCl}_{2} 2 \mathrm{mM}$, Hepes $\mathrm{pH} 7.410 \mathrm{mM}$, glucose $10 \mathrm{mM} ; 37^{\circ} \mathrm{C}$ ) and stimulated for 20s with capsaicin (Tocris0462 500ng/ml). Live-imaging was acquired by Nikon Eclipse-TE-2000U microscope (20x magnification) and analyzed by ImageJ (Fluo-4 signaling: increased fluorescence emission at 506nm).

\section{DRG culture infection and treatments}

WT and Cdk15 null DRG-neurons seeded at a density of 5,000 cells/ $/ \mathrm{cm}^{2}$ were infected with AAV9 viral particles (AAV9-Ctrl-ss-pTR-CBh-GFP-stuffer; AAV9-ss-pTR-CBh-HACDKL5 107$) 24 \mathrm{~h}$ after plating (100,000 virus genomes (VG)/cell) (66). Microscopic analysis was carried out $24 \mathrm{~h}$ post transduction with a Nikon Eclipse-TE-2000U microscope (20x magnification, bright-field phase contrast or GFP-488nm). 24, 48 and $72 \mathrm{hrs}$ after plating, the neurons were treated with KN92, KN93 $(0.5 \mu \mathrm{M}$, Tocris 4130 and 1278) or DMSO alone (67). 72 hours after the treatment or the infection, the respective efficacy of KN93 and AAV9 particles was investigated (phospho-CaMKIIa and Cdk15-protein expression). Cdkl5protein Expression Analysis: $72 \mathrm{hrs}$ after the treatment, the medium was removed and the cultures were scraped into RIPA buffer (50 mM Tris-HCl pH 7.4, $150 \mathrm{mM} \mathrm{NaCl}, 1 \%$ NP-40, $0.5 \%$ deoxycholate, protease and phosphatase inhibitors cocktails Sigma) and centrifuged at $14000 \mathrm{~g}$ for $15 \mathrm{~min}$ at $4^{\circ} \mathrm{C}$ to remove cell debris. The supernatant was transferred to a new tube, mixed with Laemmli sample buffer $(2 \mathrm{x})$, boiled at $95^{\circ} \mathrm{C}$ for $5 \mathrm{~min}$ and loaded on SDSPAGE (incubation with Cdk15 (Sigma HPA002847, 1:1000) and $\beta$ III-tubulin (Abcam ab-18207, 1:1000) antibodies). For analysis of the phospho-CaMKIIa: $72 \mathrm{hrs}$ after the treatment, the wells were fixed and processed with anti- phospho-Thr286-CaMKIIa (mouse, 
Thermo-Fisher MA1-047, 1:100) and $\beta$ III-tubulin (rabbit, abcam ab18207, 1:500)

antibodies, as previously described.

\section{GFP+ neuron sorting and RT-PCR}

For nerve injection of AAV1-CMV-GFP (Tebu-bio), after nerve exposure, 2.5 microliters of viral particles were injected with a Hamilton syringe slowly, and mice were used after 4 weeks to allow complete GFP expression. DRG were collected in Hank's balanced salt solution (HBSS) on ice and dissociated in DMEM (Invitrogen) containing $5 \mathrm{mg} / \mathrm{ml}$ Dispase II (Sigma), $2.5 \mathrm{mg} / \mathrm{ml}$ Collagenase Type II (Worthington), $1 \mathrm{mg} / \mathrm{ml}$ papain (Sigma) at $37^{\circ} \mathrm{C}$ for 30 mins with occasional mixing. Subsequently DRG were transferred to medium containing 10\% heat inactivated FBS (Invitrogen), 1X B27 (Invitrogen) in DMEM:F12 (Invitrogen) mix and were manually dissociated by pipetting to disaggregate clumps. After filtering with a 70 micron strainer, the cell suspension was mixed with a 9\% Percoll gradient (Sigma) and centrifuged at $800 \mathrm{~g} \times 12 \mathrm{~min}$ to remove tissue debris (similar to https:// www.jneurosci.org/content/27/10/2435\#sec-2). The cell suspension was run through a 130 $\mu \mathrm{m}$ nozzle at low pressure on a BD FACS Aria II machine to collect GFP positive neurons. RNA extraction was performed via Qiagen mini kit, following manufacture's protocol (as described in Arnau's paper). cDNA was synthesized from $0.5 \mu \mathrm{g}$ of total RNA using both oligodT and random hexamers from the SuperScript II Reverse Transcriptase kit (Invitrogen) and PCR was performed using $15 \mathrm{ng}$ of cDNA using the following program: 95C 5min, 30 cycles of $94: 30 \mathrm{sec} / 5430 \mathrm{sec} / 721 \mathrm{~min}$ followed by extension at $72: 10 \mathrm{~min}$.

The forward $(\mathrm{F})$ and reverse $(\mathrm{R})$ primers were:

mCdk15-Ex6-Fw, 5'-GGAGACGACCTTACGAGAGC

mCdk15-Ex6-Rv, 5'-GGACGATGTCGTTCTTGTGG

mGapdh F, 5'-AAGGTCGGTGTGAACGGATTTG

mGapdh R, 5'-GCAGTGATGGCATGGACTGTG

m Gapdh F2, 5'-GAACATCATCCCTGCATCC

mGapdh R2, 5'-CAGTGTAGCCCAAGATGCCC

mNeurofilament $\mathrm{F}$, 5'-TCAAGTGCGACGTGACGTCG

mNeurofilament R, 5'-AGTCGGTCCAACCTCACTCG

mGFAPF, 5'-CACGAAGCTAACGACTATCGC

mGFAPR, 5'-AGTGCCTCCTGGTAACTGGC

\section{DRG cultures overexpressing AIP (CaMKII inhibitor)}

Adeno-associated viral particles (AAV8) carrying pAAV-CMV-EGFP or EGFP-AIP plasmid (AIP, Autocamtide-2-related-inhibitor peptide: KKALRRQEAVDAL) were employed. WT and Cdk15 mutant null DRG-neurons were seeded at a density of 5,000 cells/cm2 and 
infected at DIV1 with AAV8 viral particles, carrying EGFP or EGFP-AIP (50,000 virus genomes (VG)/cell), and AAV9 viruses expressing GFP or human CDKL5 107 (AAV9-Ctrlss-pTR-CBh-GFP-stuffer; AAV9-ss-pTR-CBh-HA-CDKL 5 107; 50,000 virus genomes (VG)/ cell) (40). 72 hours after plating, the respective efficacy of AAV8 and AAV9 particles was investigated (phospho-CaMKIIa and CDKL5-protein expression), as previously described.

\section{Neurite outgrowth analysis}

Immunofluorescence was detected using a Nikon Eclipse-TE-2000U microscope at 20x magnification using a CDD camera (scMOS camera QImaging). Five fields per coverslip were included in the analysis. At least 30 cells per coverslip were measured. Total neurite length was measured and normalized to the number of cells. All analyses were performed blinded. Neurite analysis and measurements were performed using the Neuron-J plugin for ImageJ.

\section{Image analysis for immunohistochemistry and immunocytochemistry}

Photomicrographs were taken with Nikon Eclipse-TE-2000U microscope at 20x magnification. Alternatively, for confocal imaging, a Leica TCS SP5 II or a Zeiss LSM-780 inverted microscope ( $z$-stacks, slice spacing $1.65 \mu \mathrm{m}$ ) were used at $20 \times$ or $63 \mathrm{x}$ magnification. DRG, spinal cord and sciatic nerve micrographs were processed with the software ImageJ: a constant fluorescence intensity threshold was set across each cell (or area) in control-tissues. On the basis of this threshold, for each cell (or area) in the samples, the intensity of pixels was calculated in each channel. This was done in triplicate and the investigator was blinded to the experimental group.

\section{Single-cell calcium imaging}

We assessed changes in the intracellular $\mathrm{Ca} 2+$ concentration $\left(\left[\mathrm{Ca}^{2+}\right]_{\mathrm{i}}\right)$ using conventional ratiometric imaging in cultured DRG neurons isolated from WT and $\mathrm{CDKL}^{-/ y}$ mice. Briefly, after loading with $1 \mu \mathrm{M}$ Fura-2 (Invitrogen), the cells were exposed to capsaicin (500ng/ml) for 20 seconds and then to $50 \mathrm{mM} \mathrm{KCl} \mathrm{(20} \mathrm{seconds)} \mathrm{in} \mathrm{(in} \mathrm{mM}$ ) $\mathrm{NaCl} 160, \mathrm{KCl}$ 2.5, $\mathrm{CaCl}_{2} 1, \mathrm{MgCl}_{2} 2$, Hepes 10 , glucose $10\left(\mathrm{pH} 7.4 ; 37^{\circ} \mathrm{C}\right)$ with a flow rate of $\sim 2 \mathrm{ml} / \mathrm{min}$. Recordings, with $355 \mathrm{nM}$ and $380 \mathrm{nM}$ excitation and at $0.5 \mathrm{~Hz}$, were done with the WinFluor (J Dempster, Strathclyde University), and analyzed using the Clampfit (Invitrogen), software packages. Measurements were repeated at least in 3 independent cultures with analyzing $\sim 100$ cells in each condition. Responses of CDKL5 $5^{-/ y}$ neurons were normalized to those of WT cells (68).

\section{Intradermal injection of capsaicin}

Capsaicin $(10 \mu \mathrm{l})\left[0.1 \mathrm{~g} / \mathrm{ml}^{-1} 100 \%\right.$ ethanol; $1: 1000$ in PBS, $0.5 \%$ Tween $80,10 \%$ ethanol] or vehicle (PBS, $0.5 \%$ Tween $80,10 \%$ ethanol) was administered via intraplantar injection (69) to adult WT and Cdk15 mutant mice or to adult Cdk15-Floxed mice treated, two months before, with AAV5-GFP or AAV5-GFP-CRE (intra-sciatic injection). After capsaicin injection, the licking time was measured during the first 3 minutes. Finally, WT vs $C d k 15$ null adult mice were sacrificed to study the activation of the peripheral pain pathways in fixed sciatic DRG and spinal cord. Immunohistochemistry on DRG and spinal cord sections 
was performed by using anti-phospho-Thr286-CaMKIIa (mouse, Thermo-Fisher MA1-047, 1:100), or anti-phospho-Thr202/Tyr204-Erk1/2 (rabbit, Cell Signaling \#9101, 1:100).

\section{HEK-293 Cell Transfection, CDKL5-Immunoprecipitation and Kinase Assay}

HEK-293 cells were cultured on D-Poly-lysine in 60mm plates (DMEM Gibco, 0.7\% glucose, $10 \%$ Fetal Bovine Serum, 200mM glutamine with 1\% Penicillin/Streptomycin solution). After reaching $85 \%$ confluency, the cells were co-transfected with Lipofectamine vesicles (Thermo-Fisher L3000001 Lipofectamine-3000) containing two target DNA: pcDNA5-FRT/TO-C-3FLAG expressing rat-CAMKIIa and human-CDKL5 (or, alternatively, human-CDKL5-K42R); the control cells were treated with only Lipofectamine. The target plasmids (1ug per sample) and $15 \mu$ Lipofectamine were added to the transfection medium Opti-MEM Gibco, left at room temperature for 15 minutes, to enable the transfer of plasmids into the vesicles, and then added to the HEK cells (incubation: 4 hours at $37^{\circ} \mathrm{C}$ ). Finally, the transfection medium was replaced with definitive medium for three days. The cells were collected directly in $1 \mathrm{ml}$ of Lysis Buffer (20mM pH 7.4 Hepes, 150mM NaCl, 0.1\% NP40, PIC Sigma P8340-1x, 1mM DTT, PhosSTOP Sigma P0044-1x), sonicated $(1 \mathrm{~min})$ and then centrifugated at $10000 \mathrm{~g}\left(5 \mathrm{~min}, 4^{\circ} \mathrm{C}\right)$. The protein concentration of the supernatant was determined by the Bradford method and the CDKL5 Immunoprecipitation was performed as described ("Cdk15 Immunoprecipitation"). To confirm the overexpression of the kinases, the $5 \%$ of the supernatant was directly loaded on SDS-PAGE. The kinase activity of CDKL5 was simultaneously verified using the supernatant of the lysate from plates overexpressing CDKL5 or its inactive form CDKL5K42 (Kinase-Dead), exposed to Anti-Flag Beads (Sigma M2 Magnetic Beads; 2 hours, wheel $4^{\circ} \mathrm{C}$ ). The beads were then washed three times (wheel $4^{\circ} \mathrm{C}, 20 \mathrm{~min}$ ) in Lysis Buffer, resuspended in Kinase Buffer-1x (Thermo-Fisher PV3189) and added to a mixture of 32PATP $(1 \mathrm{mM})$ and cold-ATP $(1 \mathrm{mM})$. After incubation $\left(30\right.$ minutes at $\left.30^{\circ} \mathrm{C}\right)$, the elution was performed by using Laemmli $6 \mathrm{x}\left(30 \mu \mathrm{l}, 70^{\circ} \mathrm{C}, 3 \mathrm{~min}\right)$ and the samples were loaded on SDSPAGE. The gel, fixed in methanol $2 \%$ ( 1 hour, RT), was dried $\left(80^{\circ} \mathrm{C}, 2\right.$ hours) and exposed to an X-ray film for two days.

\section{Statistics and reproducibility}

Data are plotted as mean \pm S.D. or S.E.M. All experiments were performed in triplicate unless specified. Asterisks indicate a significant difference analyzed by ANOVA with Tukey or Sidak post hoc test, Student's $t$-test as indicated for normal distributions, parametric Twotailed test and Fisher's combined probability test. All data analysis was performed blinded to the experimental group.

\section{Supplementary Material}

Refer to Web version on PubMed Central for supplementary material.

\section{Acknowledgments}

This work was supported by start-up funds from the Division of Brain Sciences, BRC funds, Imperial College London (SDG); Wings for Life (SDG); The Rosetrees Trust (SDG); the NIHR Imperial Biomedical Research Centre (SDG); International Foundation for CDKL5 Research (HL, JD, KW) and NHMRC (HL). We would like to thank Dr. Anja Freiwald for assistance with the proteomics data analysis (proteomics facility in Mainz), Dr. Stephen 
B McMahon (Kings College London) for insightful discussions, Dr. Elisabeth Bradbury (Kings College London) for the support with the thermal preference test and Dr. Margaux Silvestre (Crick Institute London) for assistance with mouse colonies.

\section{References}

1. Scala E, et al. CDKL5/STK9 is mutated in Rett syndrome variant with infantile spasms. J Med Genet. 2005; 42:103-107. [PubMed: 15689447]

2. Kilstrup-Nielsen C, et al. What we know and would like to know about CDKL5 and its involvement in epileptic encephalopathy. Neural Plast. 2012; 2012

3. Zhou A, Han S, Zhou ZJ. Molecular and genetic insights into an infantile epileptic encephalopathy CDKL5 disorder. Front Biol (Beijing). 2017; 12:1-6. [PubMed: 28580010]

4. Wang IT, et al. Loss of CDKL5 disrupts kinome profile and event-related potentials leading to autistic-like phenotypes in mice. Proc Natl Acad Sci U S A. 2012; 109:21516-21521. [PubMed: 23236174]

5. La Montanara P, et al. Synaptic synthesis, dephosphorylation, and degradation: a novel paradigm for an activity-dependent neuronal control of CDKL5. J Biol Chem. 2015; 290:4512-4527. [PubMed: 25555910]

6. Nawaz MS, et al. CDKL5 and Shootin1 Interact and Concur in Regulating Neuronal Polarization. PLoS One. 2016; 11:e0148634. [PubMed: 26849555]

7. Ricciardi S, et al. CDKL5 ensures excitatory synapse stability by reinforcing NGL-1-PSD95 interaction in the postsynaptic compartment and is impaired in patient iPSC-derived neurons. Nat Cell Biol. 2012; 14:911-923. [PubMed: 22922712]

8. Fuchs C, et al. Loss of CDKL5 impairs survival and dendritic growth of newborn neurons by altering AKT/GSK-3beta signaling. Neurobiol Dis. 2014; 70:53-68. [PubMed: 24952363]

9. Chen Q, et al. CDKL5, a protein associated with rett syndrome, regulates neuronal morphogenesis via Rac1 signaling. J Neurosci. 2010; 30:12777-12786. [PubMed: 20861382]

10. Baltussen LL, et al. Chemical genetic identification of CDKL5 substrates reveals its role in neuronal microtubule dynamics. EMBO J. 2018

11. Munoz IM, et al. Phosphoproteomic screening identifies physiological substrates of the CDKL5 kinase. EMBO J. 2018

12. Amendola E, et al. Mapping pathological phenotypes in a mouse model of CDKL5 disorder. PLoS One. 2014; 9:e91613. [PubMed: 24838000]

13. Fuchs C, et al. Heterozygous CDKL5 Knockout Female Mice Are a Valuable Animal Model for CDKL5 Disorder. Neural Plast. 2018; 2018

14. Okuda K, et al. Comprehensive behavioral analysis of the Cdk15 knockout mice revealed significant enhancement in anxiety- and fear-related behaviors and impairment in both acquisition and long-term retention of spatial reference memory. PLoS One. 2018; 13:e0196587. [PubMed: 29702698]

15. Fehr S, et al. There is variability in the attainment of developmental milestones in the CDKL5 disorder. J Neurodev Disord. 2015; 7:2. [PubMed: 25657822]

16. Chiu IM, et al. Transcriptional profiling at whole population and single cell levels reveals somatosensory neuron molecular diversity. Elife. 2014; 3

17. Hu G, et al. Single-cell RNA-seq reveals distinct injury responses in different types of DRG sensory neurons. Sci Rep. 2016; 6

18. Bouet $\mathrm{V}$, et al. The adhesive removal test: a sensitive method to assess sensorimotor deficits in mice. Nat Protoc. 2009; 4:1560-1564. [PubMed: 19798088]

19. Schaar KL, Brenneman MM, Savitz SI. Functional assessments in the rodent stroke model. Exp Transl Stroke Med. 2010; 2:13. [PubMed: 20642841]

20. Yu P, Santiago LY, Katagiri Y, Geller HM. Myosin II activity regulates neurite outgrowth and guidance in response to chondroitin sulfate proteoglycans. J Neurochem. 2012; 120:1117-1128. [PubMed: 22191382] 
21. Latremoliere A, et al. Neuronal-Specific TUBB3 Is Not Required for Normal Neuronal Function but Is Essential for Timely Axon Regeneration. Cell Rep. 2018; 24:1865-1879 e1869. [PubMed: 30110642]

22. He Y, et al. Role of cytoplasmic dynein in the axonal transport of microtubules and neurofilaments. J Cell Biol. 2005; 168:697-703. [PubMed: 15728192]

23. Grabham PW, Seale GE, Bennecib M, Goldberg DJ, Vallee RB. Cytoplasmic dynein and LIS1 are required for microtubule advance during growth cone remodeling and fast axonal outgrowth. $\mathbf{J}$ Neurosci. 2007; 27:5823-5834. [PubMed: 17522326]

24. Roossien DH, Lamoureux P, Miller KE. Cytoplasmic dynein pushes the cytoskeletal meshwork forward during axonal elongation. J Cell Sci. 2014; 127:3593-3602. [PubMed: 24951117]

25. Murphey RK, Caruccio PC, Getzinger M, Westgate PJ, Phillis RW. Dynein-dynactin function and sensory axon growth during Drosophila metamorphosis: A role for retrograde motors. Dev Biol. 1999; 209:86-97. [PubMed: 10208745]

26. Liu XB, Murray KD. Neuronal excitability and calcium/calmodulin-dependent protein kinase type II: location, location, location. Epilepsia. 2012; 53(1):45-52.

27. Khan S, Conte I, Carter T, Bayer KU, Molloy JE. Multiple CaMKII Binding Modes to the Actin Cytoskeleton Revealed by Single-Molecule Imaging. Biophys J. 2016; 111:395-408. [PubMed: 27463141]

28. Craddock TJ, Tuszynski JA, Hameroff S. Cytoskeletal signaling: is memory encoded in microtubule lattices by CaMKII phosphorylation? PLoS Comput Biol. 2012; 8:e1002421. [PubMed: 22412364]

29. McVicker DP, Millette MM, Dent EW. Signaling to the microtubule cytoskeleton: an unconventional role for CaMKII. Dev Neurobiol. 2015; 75:423-434. [PubMed: 25156276]

30. He Y, et al. Nonmuscle myosin IIB links cytoskeleton to IRE1alpha signaling during ER stress. Dev Cell. 2012; 23:1141-1152. [PubMed: 23237951]

31. Pfister KK. Cytoplasmic dynein and microtubule transport in the axon: the action connection. Mol Neurobiol. 1999; 20:81-91. [PubMed: 10966115]

32. Furuta A, et al. Creating biomolecular motors based on dynein and actin-binding proteins. Nat Nanotechnol. 2017; 12:233-237. [PubMed: 27842063]

33. Kojundzic SL, Puljak L, Hogan Q, Sapunar D. Depression of Ca(2+)/calmodulin-dependent protein kinase II in dorsal root ganglion neurons after spinal nerve ligation. J Comp Neurol. 2010; 518:6474. [PubMed: 19882720]

34. Yan X, et al. CaMKII-Mediated CREB Phosphorylation Is Involved in Ca2+-Induced BDNF mRNA Transcription and Neurite Outgrowth Promoted by Electrical Stimulation. PLoS One. 2016; 11:e0162784. [PubMed: 27611779]

35. Price TJ, Jeske NA, Flores CM, Hargreaves KM. Pharmacological interactions between calcium/ calmodulin-dependent kinase II alpha and TRPV1 receptors in rat trigeminal sensory neurons. Neurosci Lett. 2005; 389:94-98. [PubMed: 16095822]

36. Zhang X, Daugherty SL, de WC. Groat, Activation of CaMKII and ERK1/2 contributes to the time-dependent potentiation of $\mathrm{Ca} 2+$ response elicited by repeated application of capsaicin in rat DRG neurons. Am J Physiol Regul Integr Comp Physiol. 2011; 300:R644-654. [PubMed: 21178121]

37. Frias B, Merighi A. Capsaicin, Nociception and Pain. Molecules. 2016; 21

38. Szolcsanyi J, Sandor Z. Multisteric TRPV1 nocisensor: a target for analgesics. Trends Pharmacol Sci. 2012; 33:646-655. [PubMed: 23068431]

39. Cortright DN, Krause JE, Broom DC. TRP channels and pain. Biochim Biophys Acta. 2007; 1772:978-988. [PubMed: 17467247]

40. Yu H, et al. CaMKII Controls Whether Touch Is Painful. J Neurosci. 2015; 35:14086-14102. [PubMed: 26490852]

41. Gao YJ, Ji RR. c-Fos and pERK, which is a better marker for neuronal activation and central sensitization after noxious stimulation and tissue injury? Open Pain J. 2009; 2:11-17. [PubMed: 19898681]

42. Ji RR, Gereau RWt, Malcangio M, Strichartz GR. MAP kinase and pain. Brain Res Rev. 2009; 60:135-148. [PubMed: 19150373] 
43. Cruz CD, Cruz F. The ERK 1 and 2 pathway in the nervous system: from basic aspects to possible clinical applications in pain and visceral dysfunction. Curr Neuropharmacol. 2007; 5:244-252. [PubMed: 19305741]

44. Downs J, et al. Linking MECP2 and pain sensitivity: the example of Rett syndrome. Am J Med Genet A. 2010; 152A:1197-1205. [PubMed: 20425824]

45. Baron-Cohen S, Wheelwright S. The empathy quotient: an investigation of adults with Asperger syndrome or high functioning autism, and normal sex differences. J Autism Dev Disord. 2004; 34:163-175. [PubMed: 15162935]

46. Messmer RL, Nader R, Craig KD. Brief report: judging pain intensity in children with autism undergoing venepuncture: the influence of facial activity. J Autism Dev Disord. 2008; 38:13911394. [PubMed: 18161016]

47. Minio-Paluello I, Baron-Cohen S, Avenanti A, Walsh V, Aglioti SM. Absence of embodied empathy during pain observation in Asperger syndrome. Biol Psychiatry. 2009; 65:55-62. [PubMed: 18814863]

48. Tordjman S, et al. Pain reactivity and plasma beta-endorphin in children and adolescents with autistic disorder. PLoS One. 2009; 4:e5289. [PubMed: 19707566]

49. Han Q, et al. SHANK3 Deficiency Impairs Heat Hyperalgesia and TRPV1 Signaling in Primary Sensory Neurons. Neuron. 2016; 92:1279-1293. [PubMed: 27916453]

50. Orefice LL, et al. Targeting Peripheral Somatosensory Neurons to Improve Tactile-Related Phenotypes in ASD Models. Cell. 2019; 178:867-886 e824. [PubMed: 31398341]

51. White R, et al. Cyclin-dependent kinase-like 5 (CDKL5) mutation screening in Rett syndrome and related disorders. Twin Res Hum Genet. 2010; 13:168-178. [PubMed: 20397747]

52. Mangatt M, et al. Prevalence and onset of comorbidities in the CDKL5 disorder differ from Rett syndrome. Orphanet J Rare Dis. 2016; 11:39. [PubMed: 27080038]

53. Wen Z, et al. Identification of autism-related MECP2 mutations by whole-exome sequencing and functional validation. Mol Autism. 2017; 8:43. [PubMed: 28785396]

54. Stephenson JR, et al. A Novel Human CAMK2A Mutation Disrupts Dendritic Morphology and Synaptic Transmission, and Causes ASD-Related Behaviors. J Neurosci. 2017; 37:2216-2233. [PubMed: 28130356]

55. Nagarajan RP, Hogart AR, Gwye Y, Martin MR, LaSalle JM. Reduced MeCP2 expression is frequent in autism frontal cortex and correlates with aberrant MECP2 promoter methylation. Epigenetics. 2006; 1:e1-11. [PubMed: 17486179]

56. Zhang R, et al. MeCP2 plays an analgesic role in pain transmission through regulating CREB / miR-132 pathway. Mol Pain. 2015; 11:19. [PubMed: 25885346]

57. Symons FJ, et al. Peripheral Innervation in Children With Global Developmental Delay: Biomarker for Risk for Self-Injurious Behavior? J Child Neurol. 2015; 30:1722-1727. [PubMed: 25918119]

58. Dacci P, et al. Foot pad skin biopsy in mouse models of hereditary neuropathy. Glia. 2010; 58:2005-2016. [PubMed: 20878767]

59. Timar B, et al. The usefulness of quantifying intraepidermal nerve fibers density in the diagnostic of diabetic peripheral neuropathy: a cross-sectional study. Diabetol Metab Syndr. 2016; 8:31. [PubMed: 27069510]

60. Chambers SM, et al. Combined small-molecule inhibition accelerates developmental timing and converts human pluripotent stem cells into nociceptors. Nat Biotechnol. 2012; 30:715-720. [PubMed: 22750882]

61. Chaplan SR, Bach FW, Pogrel JW, Chung JM, Yaksh TL. Quantitative assessment of tactile allodynia in the rat paw. J Neurosci Methods. 1994; 53:55-63. [PubMed: 7990513]

62. Martinez E, et al. Corticostriatal Regulation of Acute Pain. Front Cell Neurosci. 2017; 11:146. [PubMed: 28603489]

63. Hsu JL, Huang SY, Chow NH, Chen SH. Stable-isotope dimethyl labeling for quantitative proteomics. Anal Chem. 2003; 75:6843-6852. [PubMed: 14670044]

64. Rappsilber J, Mann M, Ishihama Y. Protocol for micro-purification, enrichment, pre-fractionation and storage of peptides for proteomics using StageTips. Nat Protoc. 2007; 2:1896-1906. [PubMed: 17703201] 
65. Cox J, Mann M. MaxQuant enables high peptide identification rates, individualized p.p.b.-range mass accuracies and proteome-wide protein quantification. Nat Biotechnol. 2008; 26:1367-1372. [PubMed: 19029910]

66. Valdor M, et al. RNA interference-based functional knockdown of the voltage-gated potassium channel Kv7.2 in dorsal root ganglion neurons after in vitro and in vivo gene transfer by adenoassociated virus vectors. Mol Pain. 2018; 14

67. Xu GY, Huang LY. Ca2+/calmodulin-dependent protein kinase II potentiates ATP responses by promoting trafficking of P2X receptors. Proc Natl Acad Sci U S A. 2004; 101:11868-11873. [PubMed: 15292517]

68. Srikanth S, Gwack Y. Measurement of intracellular Ca2+ concentration in single cells using ratiometric calcium dyes. Methods Mol Biol. 2013; 963:3-14. [PubMed: 23296601]

69. Murthy SE, et al. The mechanosensitive ion channel Piezo2 mediates sensitivity to mechanical pain in mice. Sci Transl Med. 2018; 10 


\section{One Sentence Summary}

Cyclin-dependent-like kinase 5 (Cdk15) controls nociception in human neurons and murine models of Cdk15 deficiency disorder via CaMKII-dependent mechanisms 

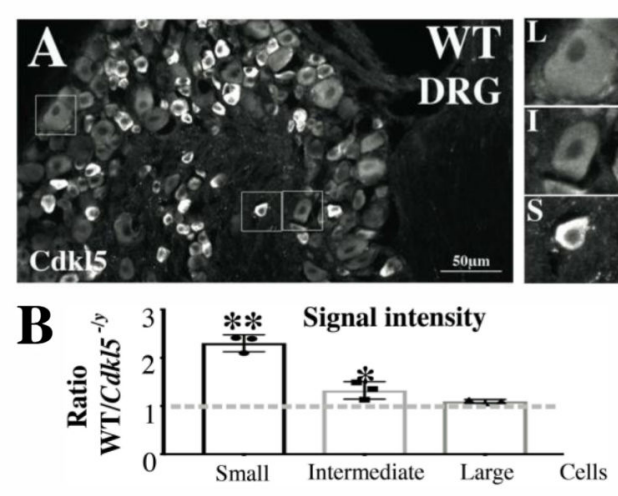

D Coexpression of Cdk15 with DRG neuronal subtypes
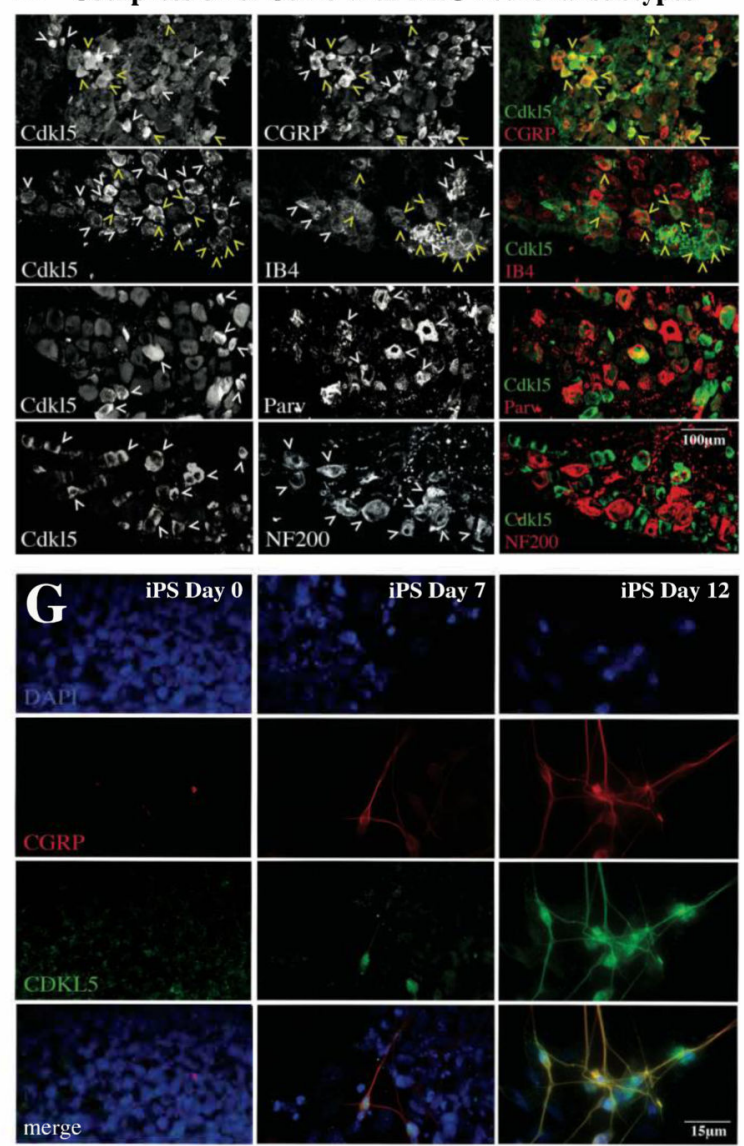
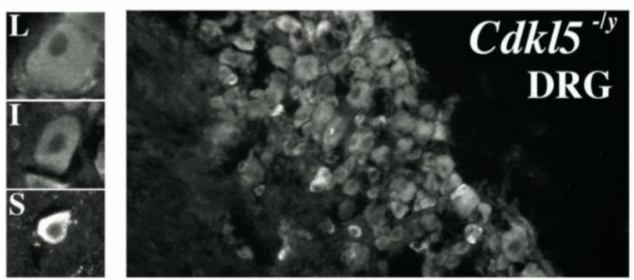

$\mathbf{C} \cong{ }_{50}^{56.25}{ }^{55}$ Size distribution

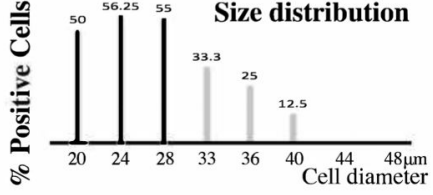

$\mathbf{E}$
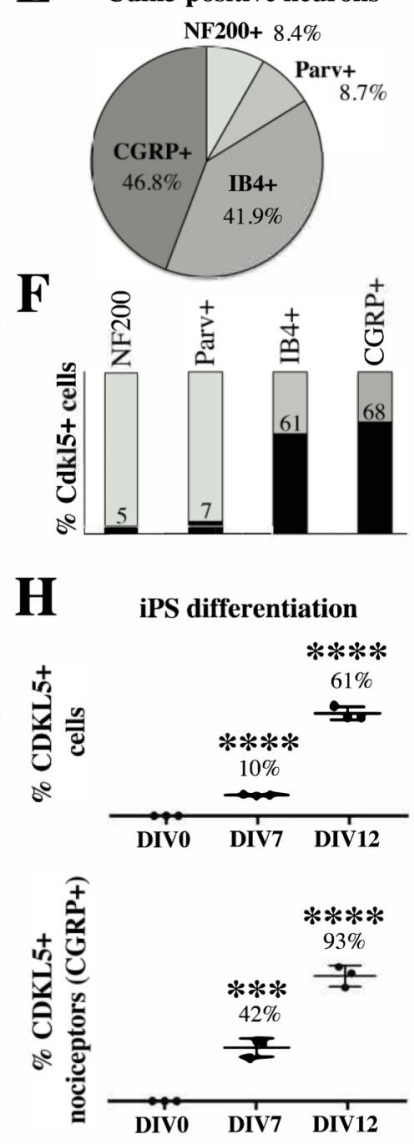

Figure 1. Cdk15 is expressed in nociceptors.

A. Cdk15-immunostaining in DRG from WT and Cdk15-/y mice (20x, Nikon EclipseTE-2000U microscope). L-I-S: insets show high magnification of large, intermediate and small diameter DRG neurons. B. Graphs showing ratio of signal intensity between WT and $C d k 15^{-/ y}$ three neuronal subpopulations. N: 3 mice each, Mean with SD, Student's $t$-test, $* * P<0.01 * P<0.05$. C. Graphs showing percentage of Cdk15+ positive neurons in the indicated sizes. N:3 mice each. D. Confocal immunofluorescence images of DRG biopsies from P70 WT mice showing the co-expression of Cdk15, CGRP, IB4, Parvalbumin and 
NF200 (20x images from Zeiss LSM-780; yellow arrowheads: co-expression; white arrow heads: expression of Cdkl5 or individual cell type markers only). E. Percentage of Cdkl5+ cells co-expressing each neuronal marker (average percentage values represent average from 3 independent mice). F. Percentage of Parvalbumin+, IB4+, CGRP+ or NF200+ cells coexpressing Cdkl5+ (average percentage values represent average from 3 independent mice). G. CDKL5 and CGRP immunostaining from isogenic iPS derived neurons after 0, 7 and 12 days of differentiation into nociceptors (63x, Leica TCS SP5II). H. Graphs showing the percentage of CDKL5+ cells and CDKL5+ neurons coexpressing CGRP at different stages of differentiation. N: 3, Mean with SD, One-way ANOVA, Tuckey's post-hoc, $* * * *$ $P<0.0001 * * * P<0.001$. 


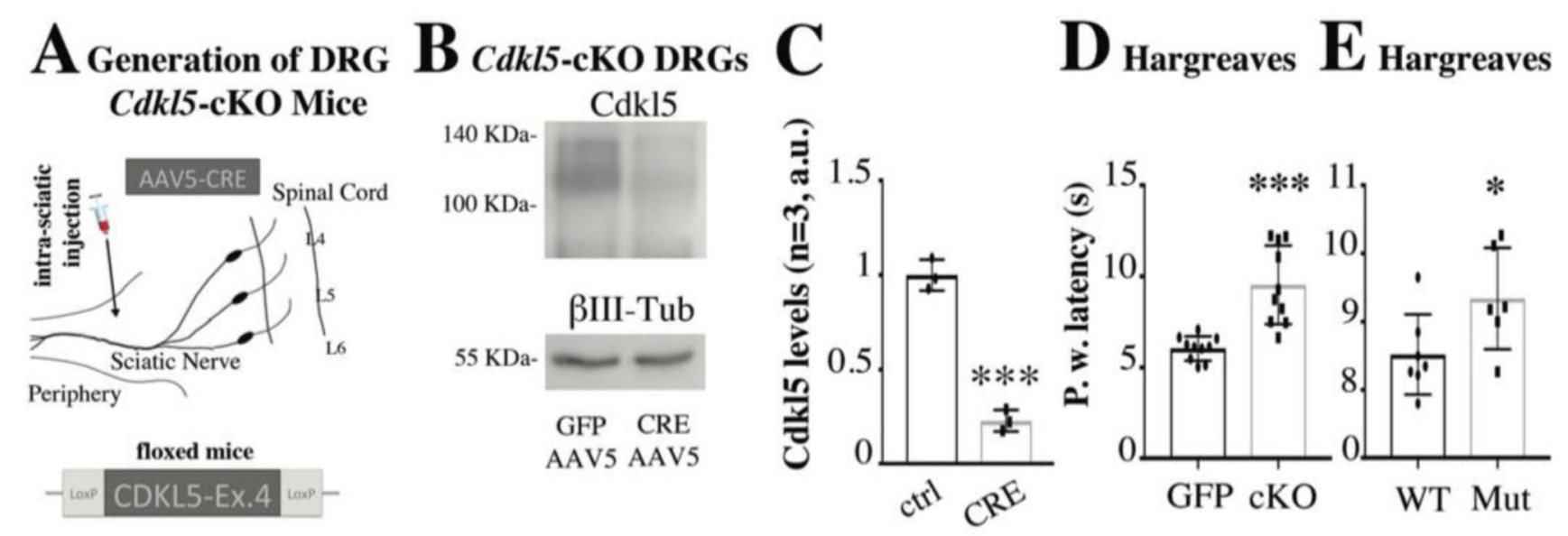

F

von Frey $G$ von Frey

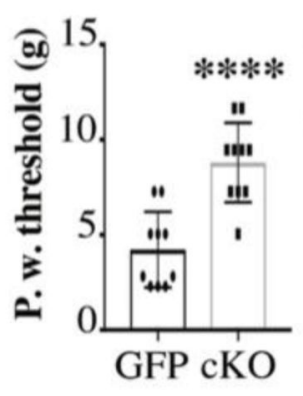

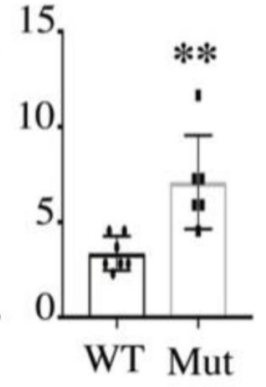

$\mathbf{H}$

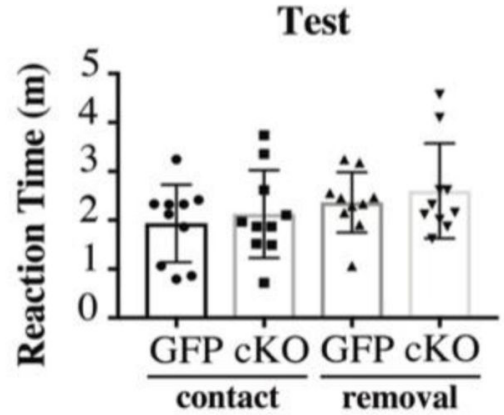

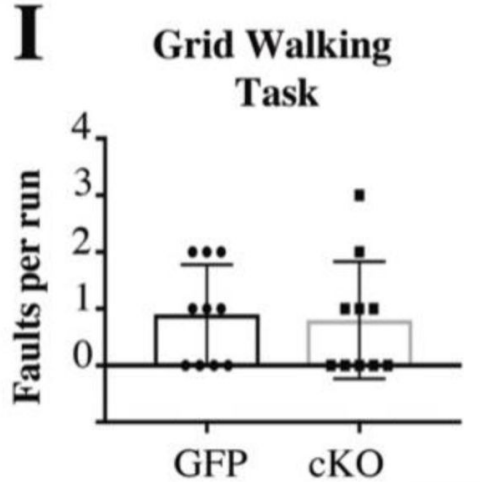

Figure 2. Conditional knockdown of Cdkl5 impairs nociception.

A. Schematic illustrating conditional knockdown of Cdk15 in DRG neurons. Depicted is the injection of AAV5 particles into the sciatic nerve of 12 weeks old $C d k 15$-floxed mice. B. Immunoblotting showing strongly reduced expression of Cdk15 from bilateral sciatic DRG (pool of 4 mice) 8 weeks after AAV5-GFP or AAV5-GFP-cre. $\beta$ III-tubulin has been used as loading control. C. Graph showing the conditional knockdown of Cdk15 in DRG (Cdk15floxed mice) after GFP-AAV5 vs CRE-AAV5 injection in the sciatic nerve ( $N=3$, Mean with S.E.M., Student's t-test, $* * * P<0.001)$. D-G. Behavioral assessment of pain sensitivity with Hargreaves (thermal nociception; Paw withdrawal latency) and von Frey (mechanical allodynia; Paw withdrawal threshold) shows significantly reduced sensitivity in Cdk15 conditionally deleted $(\mathbf{E}, \mathbf{F})$ as well as in $C d k 15^{-/ y}$ mice $(\mathbf{E}, \mathbf{G})$. D, F. N:10 mice, Mean with SD, Student's $t$-test, $* * * * P<0.0001 * * * P<0.001$. E,G. N: 7 WT vs 6 Mut mice, Mean with $\mathrm{SD}$, Student's $t$-test, $* * P<0.01 * P<0.05$. H. Graph showing no difference in the reaction time (time to contact and time to remove the adhesive tape from the paw) in Cdkl5 DRG conditionally deleted (cKO) vs control GFP injected mice. N:10, Mean with SD, Student's ttest, $P>0.05$. I. Graph showing no difference in the number of posterior foot slips in each run on a Gridwalk in Cdk15 DRG conditionally deleted (cKO) vs control GFP injected mice. N: 10 mice per group, Mean with SD, Student's t-test, $P>0.05$. 


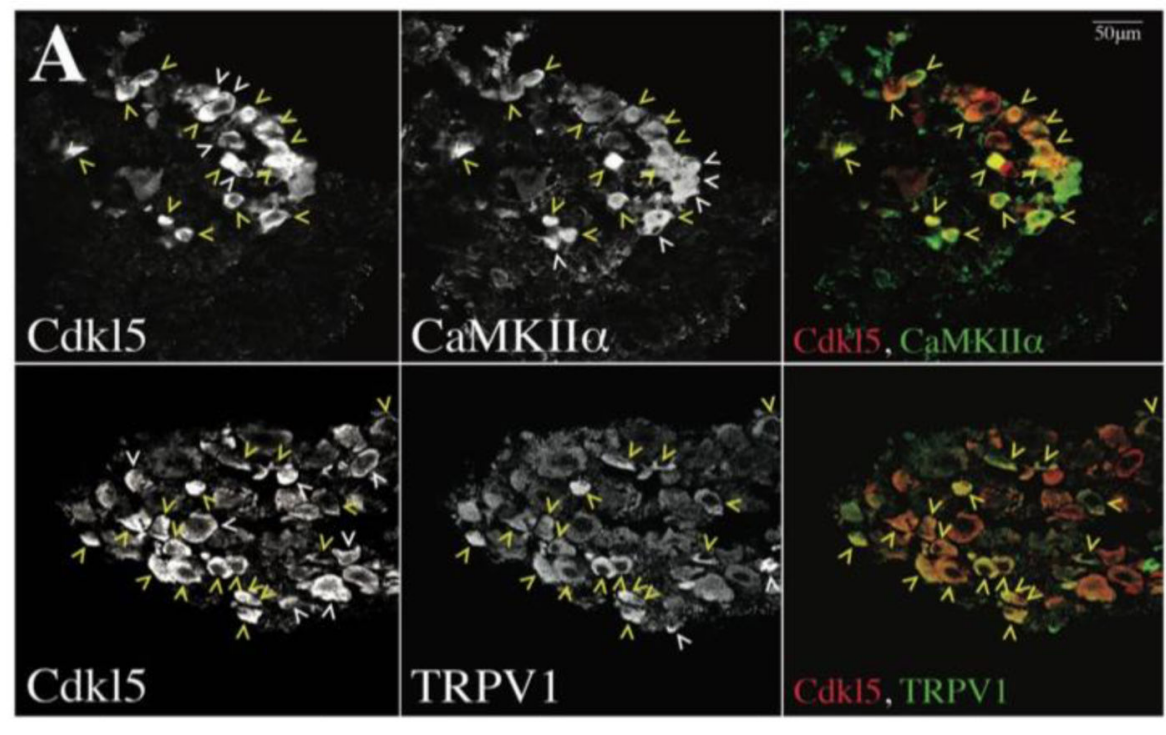

D Cdkl5 and CaMKII $\alpha$ are in close molecular proximity in DRGs
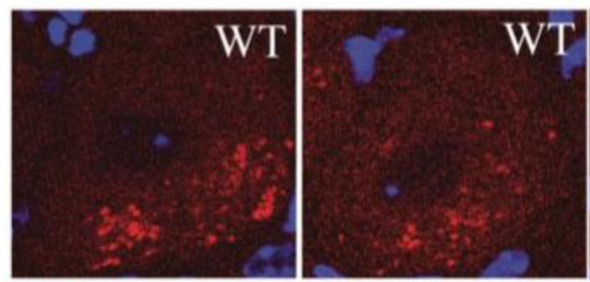

WT

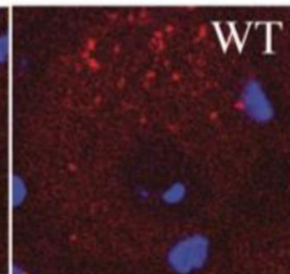

PLA signal, DAPI

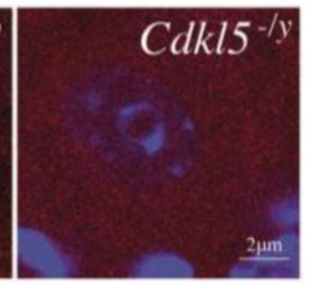

\begin{tabular}{|l|c|c|}
\hline B & $\begin{array}{c}\text { Cell diameter } \\
\text {-Average- }\end{array}$ & $\begin{array}{c}\text { Cell diameter } \\
\text {-Range- }\end{array}$ \\
\hline Cdkl5+ & $25.18+/-4.6 \mu \mathrm{m}$ & $17.8-38 \mu \mathrm{m}$ \\
\hline CaMKIIa+ & $26.34+/-4.66 \mu \mathrm{m}$ & $20.6-40.5 \mu \mathrm{m}$ \\
\hline TRPV1+ & $25.23+/-4.7 \mu \mathrm{m}$ & $15.3-38.1 \mu \mathrm{m}$ \\
\hline
\end{tabular}
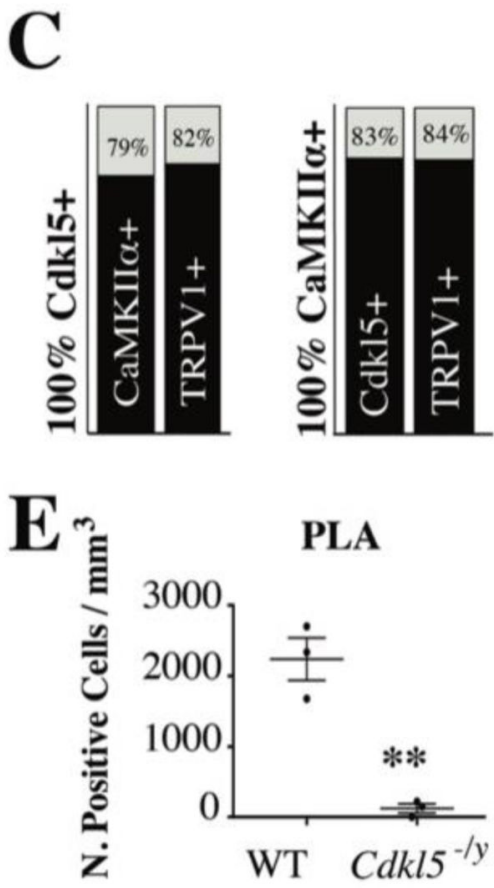

Figure 3. Coexpression of Cdkl5 with CaMKIIa and TRPV1.

A. Confocal microscopy of immunofluorescence showing co-expression of Cdk15, CaMKIIa and TRPV1 (magnification 20x, Leica TCS SP5II; yellow arrowheads: coexpression; white arrowheads: expression of Cdkl5 or individual cell type markers only). B. Average diameter and SD of each Cdk15+, CaMKIIa+ or TRPV1+ population of cells (average values from 3 independent mice). C. Percentage of Cdkl5+ or CaMKIIa+ cells coexpressing each marker (average percentage values represent average from 3 independent mice. Cdkl5+ cells coexpressing CaMKIIa: 79.07+/-1.56\%; Cdkl5+ cells coexpressing TRPV1: 82.3+/-6.8\%; CaMKIIa+ cells coexpressing Cdk15: 83.97+/-3.44\%; CaMKIIa+ cells coexpressing TRPV1: 84.01+/-5.66\%. D. Confocal images showing individual WT or Cdk15-/y DRG neurons (63x, Leica TCS SP5II) after proximity ligation assay (PLA) (red) and DAPI staining (blue). The presence and intensity of red dots represent the presence and degree of molecular proximity between Cdkl5 and CaMKIIa. E. Graph showing the number of PLA-positive cells in WT and $C d k 15^{/ y}$ DRGs. N: 3 animals, Mean with SEM, Student's $t$-test, $* * P<0.005$. 
A
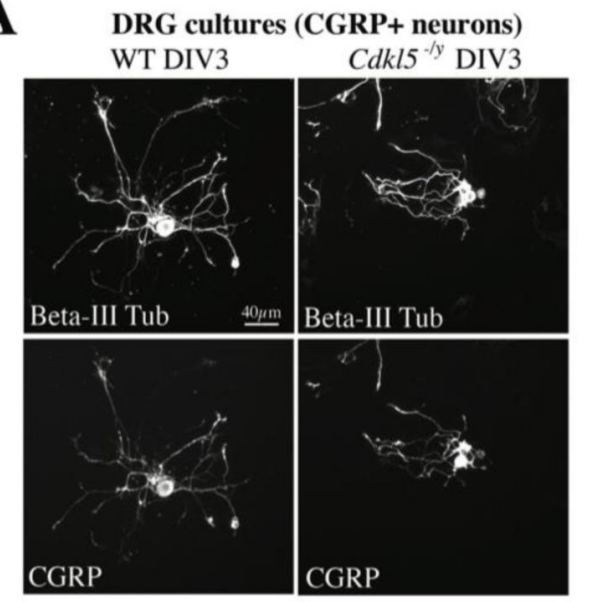

C

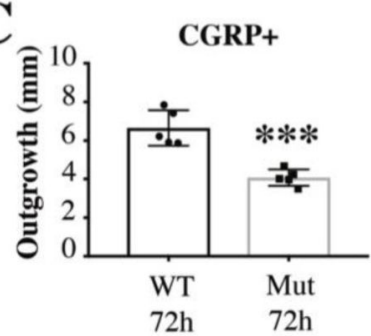

F

iPS-derived CGRP+ Cells
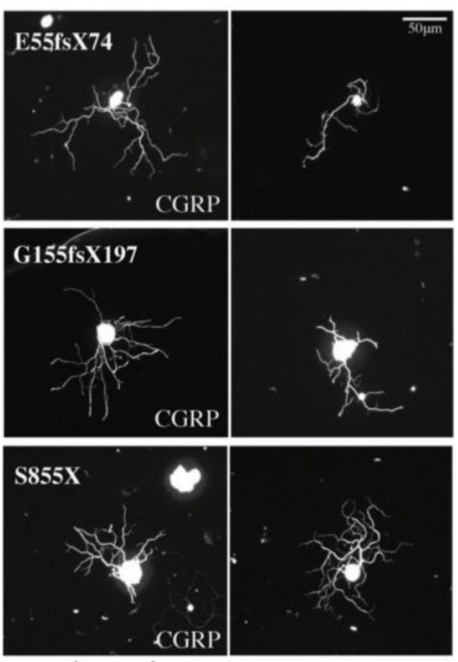

isogenic
D

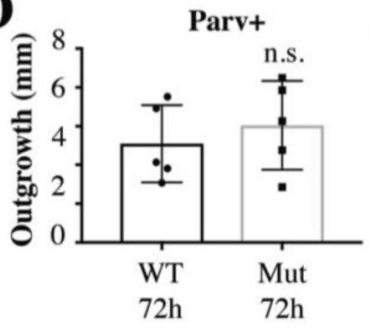

G Neurite outgrowth
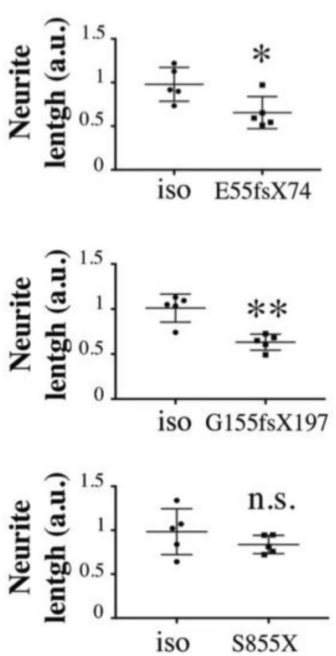

E
B DRG cultures (Parv+ neurons)
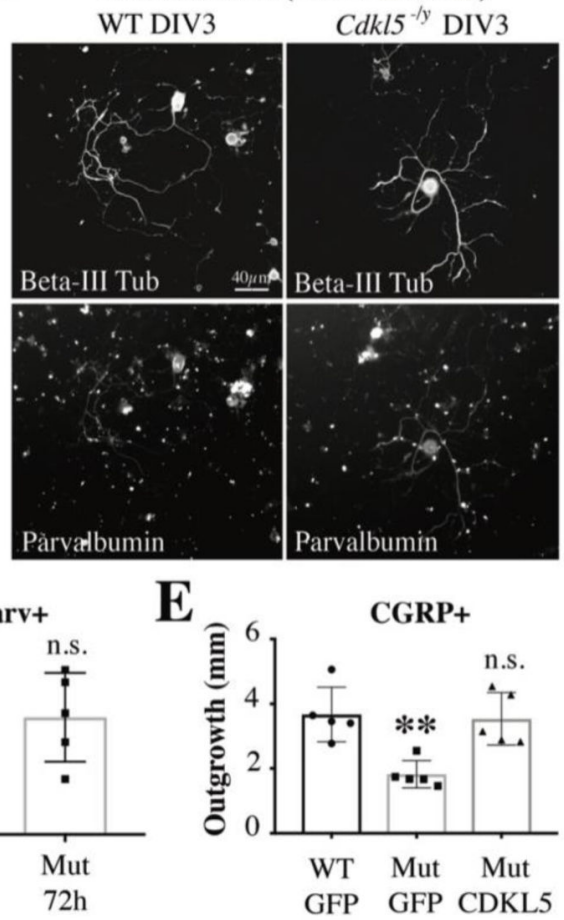

H CDKL5 mutations

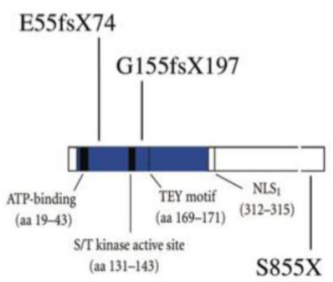

Mutations Missing domains

E55fsX74 Kinase Active Site,

TEY, NLS1, NLS2, NES

G155fsX197 TEY, NLS1, NLS2, NES

5855X terminal tail

Figure 4. Cdkl5 is required for outgrowth of human sensory neurons.

A,B. Representative immunofluorescence of $\beta$ III-tubulin, CGRP or Parvalbumin in WT and Cdk15 ${ }^{-/ y}$ DRG cultured neurons (DIV3) showing reduced neurite outgrowth in CGRP+ but nor Parv $+C d k 15^{-/ y}$ neurons. C. Graph showing reduced neurite outgrowth in CGRP+ neurons from $C d k 15^{-/ y}$ mice (N:5 biological replicates, Mean with SD, Student's $t$-test, $* * * P<0.001)$ that is rescued by CDKL5 overexpression $(\mathbf{E} ; \mathrm{N}: 5$ biological replicates, Mean with SD, One-way ANOVA, Tukey's post-hoc, ${ }^{* *} P<0.005$ ). D. Graph showing equal neurite outgrowth in Parv+ neurons from WT and $C d k 15^{-/ y}$ mice. N: 5 biological replicates, Mean 
with SD, Student's $t$-test, $P>0.05$. F. Representative images of CGRP immunostaining of iPS derived sensory neurons from three CDKL5 patients carrying unique mutations (E55fsX74; G155fsX197; S855X), compared to their isogenic controls (20x images, Nikon TE-2000U fluorescence microscope). G. CGRP immunostaining was used to measure total average neurite outgrowth (NeuronJ) of nociceptive neurons. N: 5 independent experiments, total neurite outgrowth/cell. Mean with SD, Student's $t$-test, $* P<0.05 * * P<0.01$. H. Schematic representation of human CDKL5 protein (adapted, (2) with the catalytic domain in blue (ATP-binding site, aa 19-43; kinase active site, aa 131-143; TEY motif, aa 169-171) and the C-terminal tail in white (Nuclear Localization Signal 1, aa 312-315; Nuclear Localization Signal 2, aa 784-789; Nuclear Export Signal, aa 836-845). 
A DRGs cultures (DIV3 neurons)

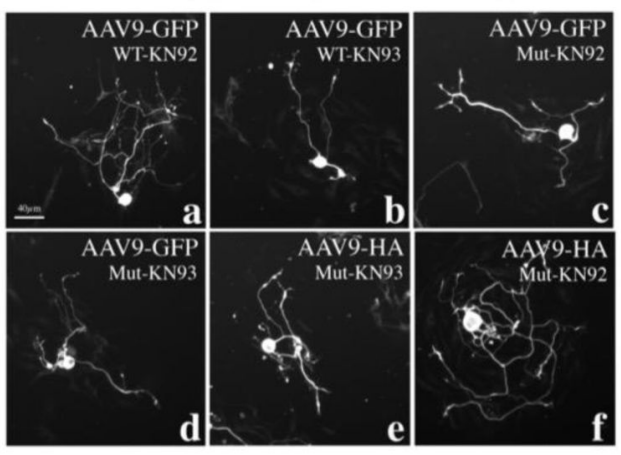

B

Neurite Outgrowth

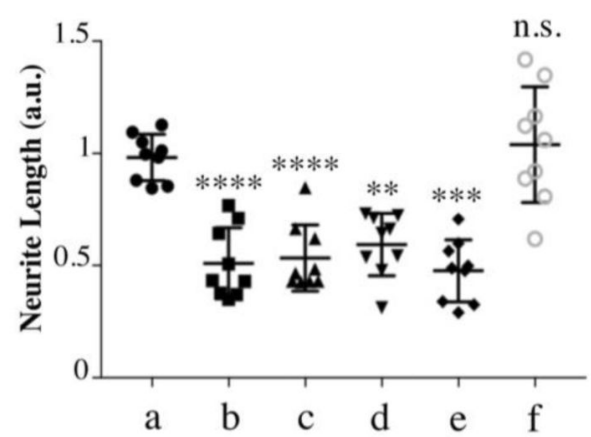

C

Calcium Imaging (Capsaicin 20s): iPS derived Nociceptors
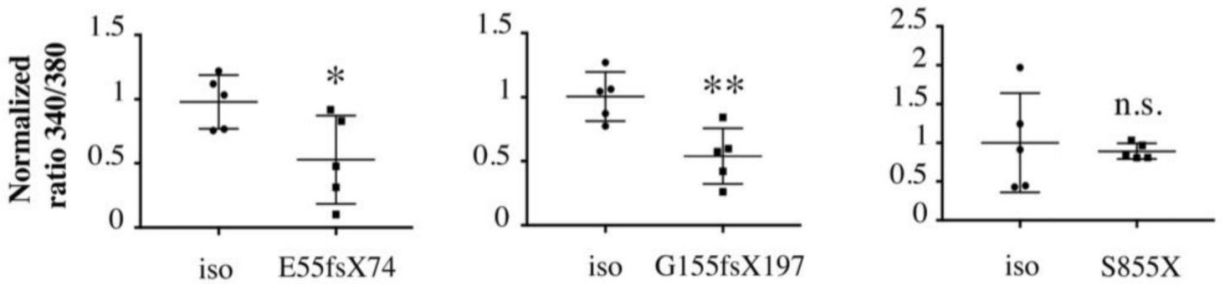

D

Calcium Signal, DRG Neurons

$\mathbf{E}$

DRG Neurons

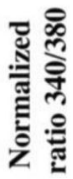
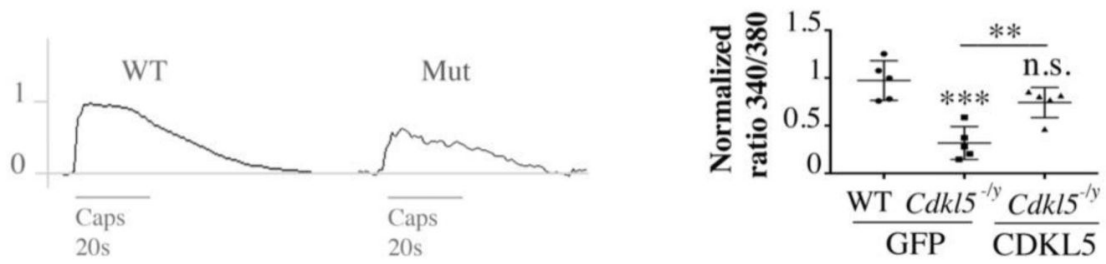

$\mathbf{F}$

DRG Neurons

G

DRG Neurons

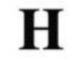

DRG Explants
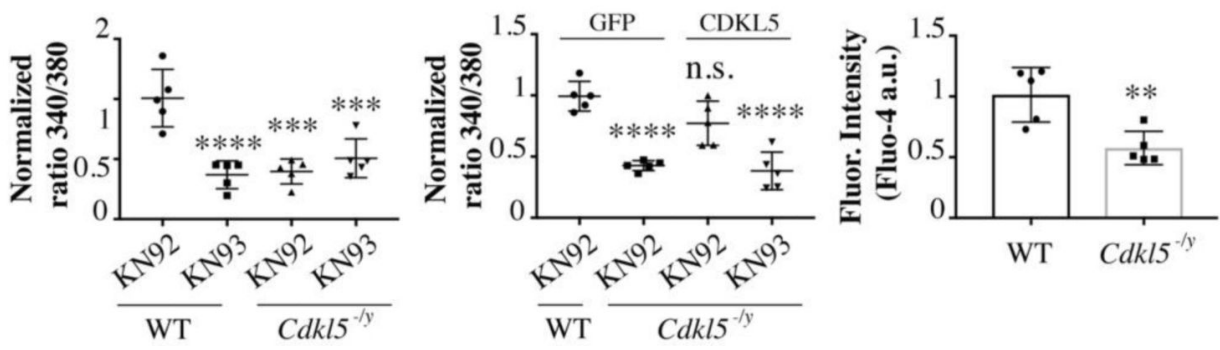

Figure 5. Cdkl5/CaMKII signaling is required for capsaicin signaling and outgrowth of sensory neurons.

A. Representative immunofluorescence of DRG cultured neurons (DIV3, from P70 WT and Cdk15 $/ y$ mice), immunostained for GFP or HA after neurons had been infected with AAV9 particles expressing GFP or HA-CDKL5 107 at DIV1 (20x, Nikon TE-2000U fluorescence microscope). DRG neurons were also treated with $0.5 \mu \mathrm{M}$ of the CaMKII inhibitor KN93 or its inert structural analog KN92. B. Graphs showing neurite outgrowth of GFP or HA positive cells that was normalized to the ctrl WT-GFP. N: 9, biological replicates, Mean with 
SD, One-way ANOVA, Tukey's post-hoc, **** $P<0.0001 * * * P<0.001 * * P<0.005$. C. Calcium imaging (Fura-2) from iPS derived nociceptors carrying three different CDKL5 mutations (E55fsX74; G155fsX197; S855X), compared with the respective isogenic controls. Graphs showing quantification of intracellular calcium in response to capsaicin. E55fsX74 and G155fsX197 neurons show reduced calcium influx after capsaicin. N: 5, average single cell Fura-2 normalized signal/well ( $\sim 30$ cells per well). Mean with SD, Student's $t$-test, $* * P<0.01 * P<0.05$. D. Representative transient traces of the calcium amount in DIV3 WT and Cdk15-/y neurons (Fura-2 tracks). E-G. Calcium imaging in cultured DRG neurons (Fura-2). DIV3 Cdk15 $5^{-/ y}$ neurons showing reduced calcium influx after capsaicin and rescue to WT amount after overexpression of CDKL5 (sspTR-CBh-HA-CDKL5107 vs GFP AAV9 particles, infection at DIV1) (E). The same experimental conditions as (D) after pre-treatment with KN93 or KN92 in WT and Cdk15 $-/ y$ neurons (F), overexpressing CDKL5 or GFP $(\mathbf{G})$. N: 5, average single cell Fura-2 normalized signal /well ( $\sim 30$ cells per well), Mean with SD, One-way ANOVA, Tukey's post-hoc, **** $P<0.0001 * * * P<0.001$

$* * P<0.005$. H. Calcium imaging in DRG-explants from WT and $C d k 15^{-/ y}$ mice. Graphs showing quantification of intracellular calcium in response to capsaicin (Fluo-4). N: 5, average single cell Fura-4 normalized signal /well ( $\sim 30$ cells per well), Mean with SD, Student's $t$-test, $* * P<0.01$. 

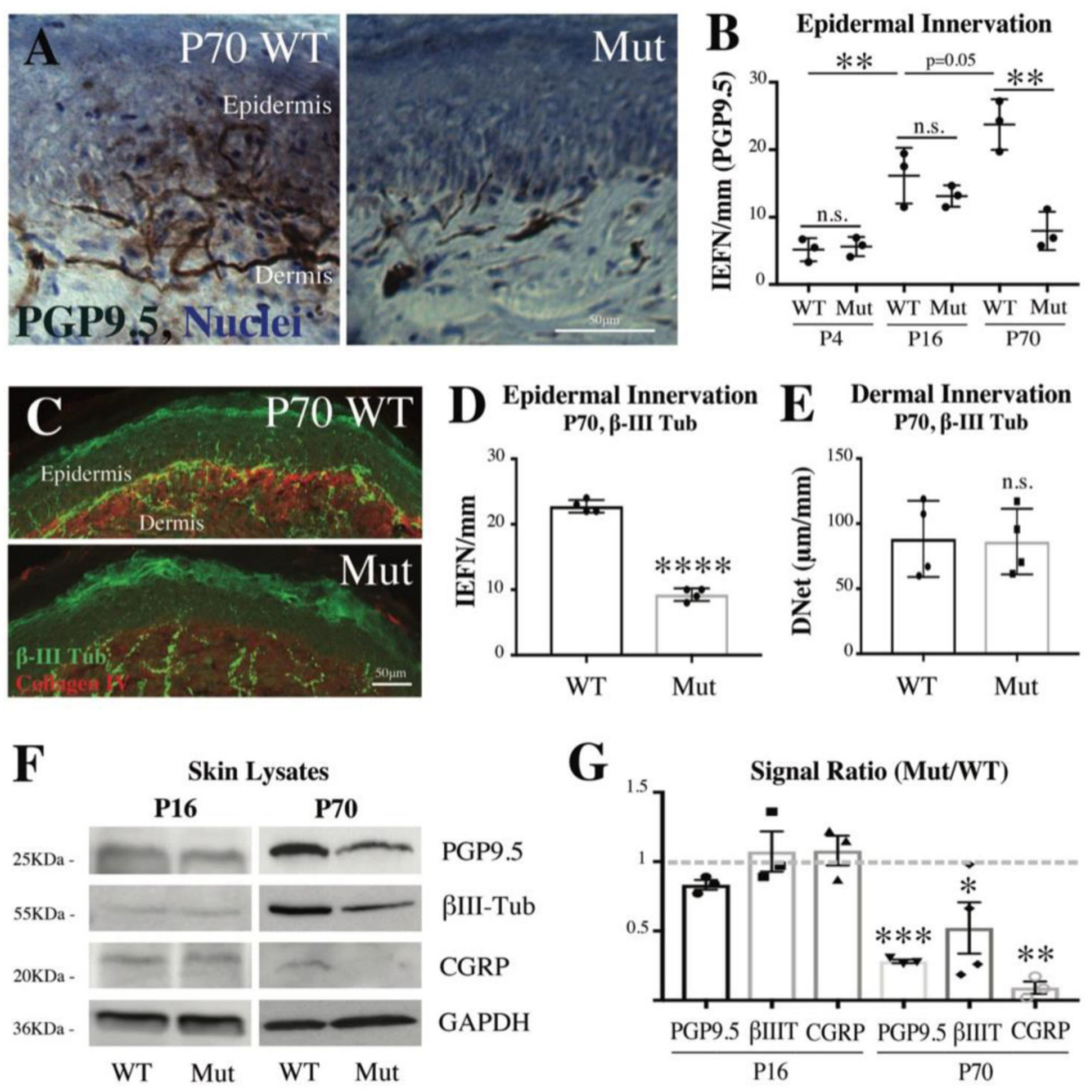

H Sciatic Nerve Lysates
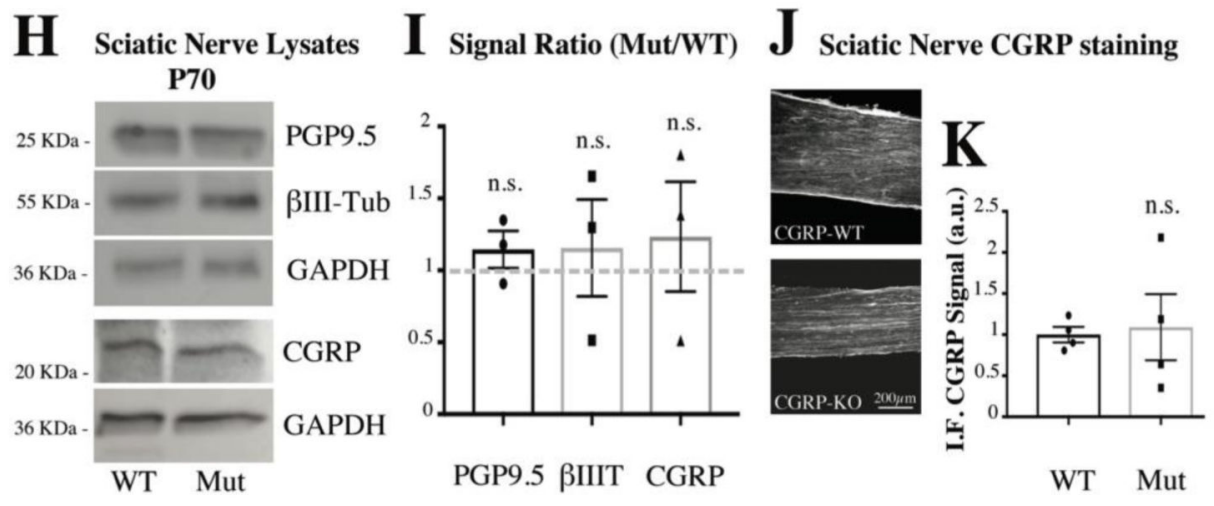

Figure 6. Cdkl5 is required for epidermal innervation.

A. Immunohistochemical localization of Nerve Fibers in the Derm and in the Epiderm of 50 $\mu \mathrm{m}$ murine skin sections (P70), using PGP9.5 as specific axonal marker (20x magnification, Zeiss LSM-780 inverted confocal microscope). Sections were counterstained for nuclei in Mayer's Haematoxylin. B. The density of the intra-epidermal nerve fibers (IEFN/mm), crossing the dermal-epidermal junction, was analyzed in WT and Cdkl5 $5^{-/ y}$ mice at different developmental stages (P4, P16, P70). N:3, Mean with SD, Two-way ANOVA, Sidak's posthoc, $* * \mathrm{P}<0.005$. C. Immunofluorescence of skin biopsies from $\mathrm{P} 70 \mathrm{WT}$ and $\mathrm{Cdk15} / \mathrm{y}$ mice. 
Representative micrographs where collagen IV identifies the epidermal basal lamina and $\beta$ III-tubulin identifies intra-epidermal nerve fibers (20x magnification, Zeiss LSM-780 inverted confocal microscope). D. Individual IENF crossing the dermal-epidermal junction were analyzed. N:4, Mean with SD, Student's t-test, $* * * * \mathrm{P}<0.0001$. E. The intra-dermal neuronal network (DNet, $\mu \mathrm{m} / \mathrm{mm}$ ) was measured by using Neuron-J software (magnification 20x). N:4, Mean with SD, Student's t-test, $P>0.05$. F. Western blotting showing the expression of neuronal markers (PGP9.5, $\beta$ III-tubulin and CGRP) from skin lysates of P16 and P70 WT and $\mathrm{Cdk15} 5^{-/ y}$ mice. G. The immunoblotting bands have been quantified by densitometry after normalization with GAPDH used as internal loading control. N: 3-4 independent experiments, Mean with SD, Two-way ANOVA, Sidak's post-hoc, $* * * * \mathrm{P}<0.0001 * * \mathrm{P}<0.005$. H. Western blotting showing the expression of PGP9.5, $\beta \mathrm{III}-$ tubulin and CGRP from sciatic nerve lysates in WT and Cdk15 $5^{-/ y}$ mice. GAPDH was used as loading control. I. Densitometry of immunoblotting bands showing no difference between P70-WT and Cdk15 $5^{-/ y}$ mice was performed after normalization to GAPDH. N: 3 independent experiments, Mean with SD, Student's t-test, P>0.05. J. Representative CGRP immunofluorescence of sciatic nerve sections from P70-WT and Cdk15-/y mice (10x, Nikon Eclipse-TE-2000U microscope). K. Graphs showing quantification of the immunofluorescence signal display no difference between WT and Cdk15 $5^{-/ y}$ mice. N: 3 mice per group, Mean with SD, Student's t-test, $\mathrm{P}>0.05$. 

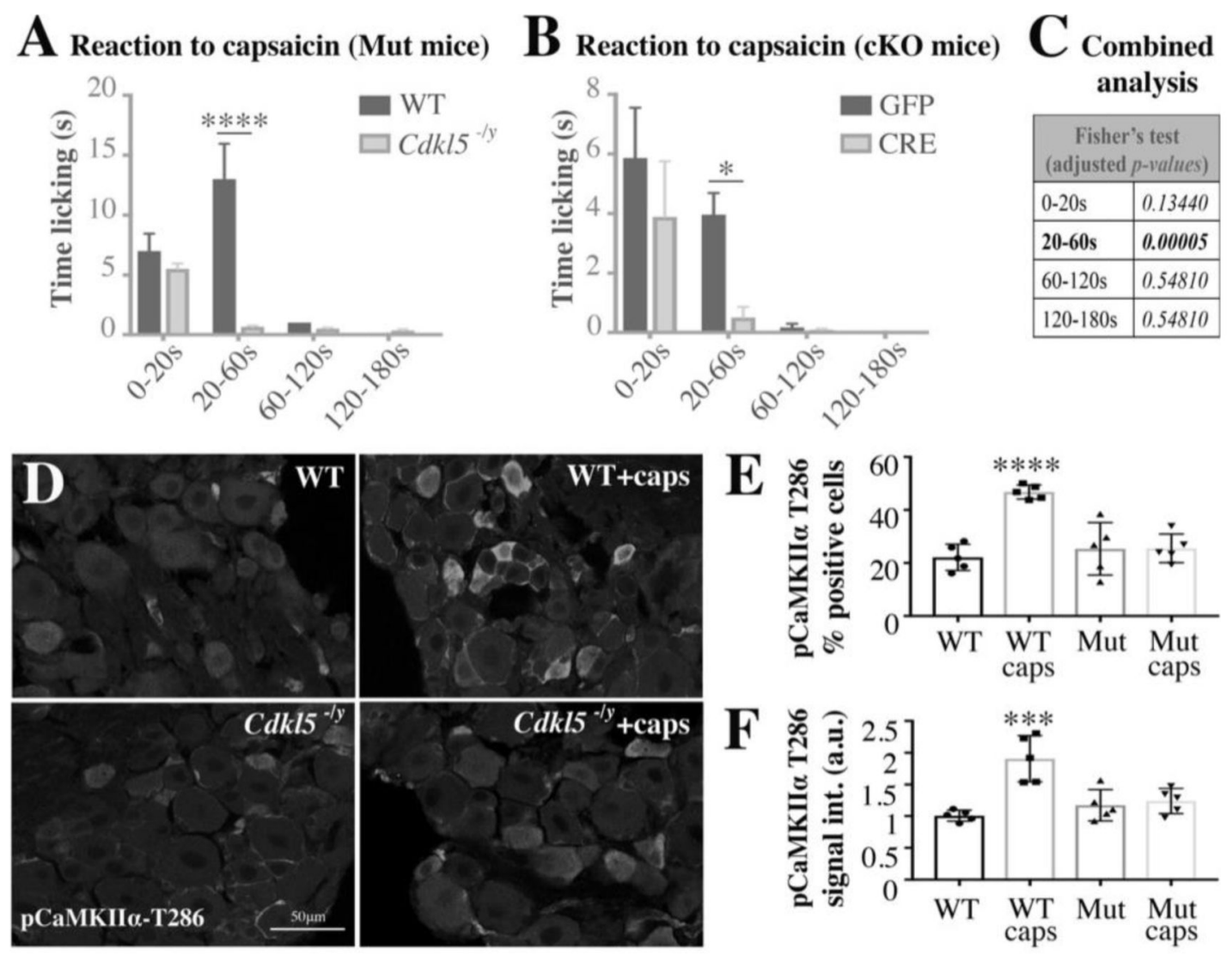
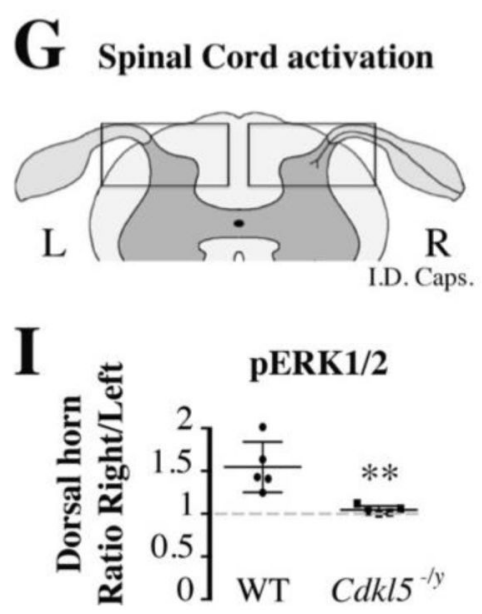
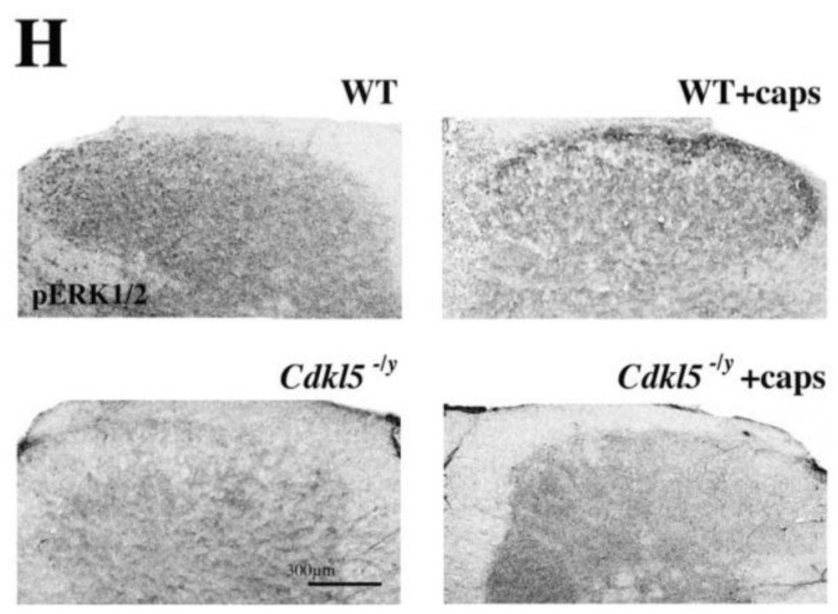

Figure 7. Cdkl5 deletion impairs capsaicin mediated nociceptive signaling and licking behavior. A,B. Bar graphs showing the time spent licking the right paw after the injection of $1 \mu \mathrm{g}$ of capsaicin in the posterior right paw vs vehicle injected in the left paw (A: WT vs $C d k 15^{-/ y}$; B: GFP vs CRE AAV5 intra-sciatic injected $C d k 15$-floxed mice). Time licking the right paw is significantly reduced in $C d k 15^{-/ y}$ mice and in mice where $C d k 15$ was conditionally deleted (cKO) in DRG. A. N: 5 mice each group, Mean with SEM, Two-way ANOVA, Sidak's posthoc, $* * * * P<0.0001$. B. N: 8 mice each group, Mean with SEM, Two-way ANOVA, Sidak's post-hoc, $* P<0.05$. C. Fisher's combined probability test (false discovery rate, meta-analysis 
adjusted for multiple testing) of the p-values from the time courses in A and B (one statistical test for each time point). D. Representative confocal immunofluorescence images for phospho-T286-CaMKIIa in WT and Cdk15 ${ }^{-/ y}$ sciatic left and right DRG (63x, Leica TCS SP5 II). Tissue was fixed immediately after the capsaicin injection test in the right paw (vehicle in the left). E, F. Bar graphs showing the percentage number of positive pCaMKIIa cells (E) and the pCaMKIIa average signal intensity in WT vs $C d k 15^{-/ y}$ left and right DRGsmall cells after normalization to WT (F). N: 5 mice per group, Mean with SD, One-way ANOVA, Tukey's post-hoc, $* * * * P<0.0001 * * * P<0.001$. G. Schematic illustrating a cross section of the lumbar spinal cord emphasizing the left (WT and Mut) and right (WT and Mut) dorsal horns (after injection of vehicle in the left paw and capsaicin in the right paw) where nociceptive neurons make a synapse in the lamina I-II (L: left, R: right). $\mathbf{H}$. Representative confocal immunofluorescence images for pERK1/2 in WT and Cdk15-/y spinal dorsal horns (10x, Leica TCS SP5 II). Tissue was fixed five minutes after capsaicin injection in the posterior right paw vs vehicle injected in the left paw. I. Graph showing lack of pERK1/2 activation (signal intensity) in spinal lamina I-II (right side vs left side) in Cdk15 $-/ y$ mice vs WT. N: 5 mice per group, Mean with SD, Student's $t$-test, $* * P<0.01$. 
Table 1

Associations between pain sensitivity, gender, age group and mutation type in 202 individuals with confirmed CDKL5 mutation.

\begin{tabular}{|c|c|c|c|c|c|c|c|c|c|}
\hline & & Normal & Reduced & \multicolumn{2}{|c|}{ Reduced versus Normal } & \multirow[t]{2}{*}{ Enhanced } & \multicolumn{2}{|c|}{ Enhanced versus Normal } & \multirow{2}{*}{$\begin{array}{c}\begin{array}{c}\text { Reduced } \\
\text { versus } \\
\text { Enhanced }\end{array} \\
\begin{array}{c}\text { Within } \\
\text { category }\end{array}\end{array}$} \\
\hline & & & & $\begin{array}{l}\text { Within } \\
\text { category }\end{array}$ & $\begin{array}{l}\text { Between } \\
\text { category }\end{array}$ & & $\begin{array}{l}\text { Within } \\
\text { category }\end{array}$ & $\begin{array}{l}\text { Between } \\
\text { category }\end{array}$ & \\
\hline & $\mathrm{N}$ & $\mathrm{n}(\%)$ & $\mathrm{n}(\%)$ & $\mathrm{RR}(95 \% \mathrm{Cl})$ & $\operatorname{RRR}(95 \% \mathrm{Cl})$ & $\mathrm{n}(\%)$ & $\mathrm{RR}(95 \% \mathrm{Cl})$ & $\operatorname{RRR}(95 \% \mathrm{Cl})$ & $\begin{array}{l}\mathrm{RR}(95 \% \\
\mathrm{Cl})\end{array}$ \\
\hline Totol & 202 & $\begin{array}{c}108 \\
(53.5)\end{array}$ & $70(34.7)$ & $\begin{array}{c}0.65 \\
(0.48,0.88)\end{array}$ & $\mathrm{n} / \mathrm{a}$ & $24(11.9)$ & $\begin{array}{c}0.22 \\
(0.14,0.35\}\end{array}$ & $\mathrm{n} / \mathrm{a}$ & $\begin{array}{c}2.92 \\
(1.83,4.64)\end{array}$ \\
\hline \multicolumn{10}{|l|}{$\begin{array}{l}\text { Age group } \\
\text { (year) }\end{array}$} \\
\hline $0-2 y$ & 82 & $\begin{array}{c}54(65 . \\
9)\end{array}$ & $21(25.6)$ & $\begin{array}{c}0.39 \\
(0.23,0.064)\end{array}$ & Ref & $7(8.5)$ & $\begin{array}{c}0.13 \\
(0.06,0.23)\end{array}$ & Ref & $\begin{array}{c}3 \\
(1.28 .7 .06)\end{array}$ \\
\hline $3-5 y$ & 45 & $\begin{array}{c}23 \\
(51.1)\end{array}$ & $16(35.6)$ & $0.70(0.37,1.32)$ & $\begin{array}{c}1.79 \\
(0.79,4.03)\end{array}$ & $6(13.3)$ & $\begin{array}{c}0.26 \\
(0.11,0.64)\end{array}$ & $\begin{array}{c}2.01 \\
(0.61,0.65)\end{array}$ & $\begin{array}{c}2.67 \\
(1.04 .6 .31)\end{array}$ \\
\hline $6+y$ & 75 & $31(41.3)$ & $33(44.0)$ & $3.06(0.65 .1 .73)$ & $2.74(1.36 .5 .53)$ & $11(14.7)$ & $\begin{array}{c}0.33 \\
(0.17 .0 . \mathrm{G} 6)\end{array}$ & $2.74(0.96 .7 .79)$ & $\begin{array}{c}3 \\
(1.52 .5 .54)\end{array}$ \\
\hline \multicolumn{10}{|l|}{ Gender } \\
\hline Female & 172 & $\begin{array}{c}93454- \\
11 .\end{array}$ & $60(14.3)$ & $\begin{array}{c}0.65 \\
(0.47,0.89)\end{array}$ & Ref & $19(11.1)$ & $0.20(0.12,0.33)$ & Ref & $\begin{array}{c}3.16 \\
(1.83 .5 .29)\end{array}$ \\
\hline Male & 30 & $\begin{array}{c}15 \\
(50.0)\end{array}$ & $10(33.3)$ & $0.67(0.30,1.48)$ & $1.03(0.44,2.45)$ & $5(16.7)$ & $\begin{array}{c}0.33 \\
(0.12,0.92)\end{array}$ & $1.63(0.53,5.03)$ & $\begin{array}{c}2 \\
{[0.68 .5 .85)}\end{array}$ \\
\hline \multicolumn{10}{|l|}{$\begin{array}{l}\text { Mutation } \\
\text { type }\end{array}$} \\
\hline $\begin{array}{l}\text { Trunratiwiu } \\
\text { after aal72 }\end{array}$ & 79 & $\begin{array}{c}48 \\
(60.5)\end{array}$ & $23(29.1)$ & $\begin{array}{c}0.48 \\
(0.29,0.79)\end{array}$ & Ref & $8(10.1)$ & $\begin{array}{c}0.17 \\
(0.08,0.35)\end{array}$ & Ref & $\begin{array}{c}2.88 \\
(1.29 .6 .42)\end{array}$ \\
\hline $\begin{array}{l}\text { No } \\
\text { functional } \\
\text { protein }^{*}\end{array}$ & 123 & $\begin{array}{c}60 \\
(48.8)\end{array}$ & $47(38.2)$ & $\begin{array}{c}0.78 \\
(0.53,1.15)\end{array}$ & $\begin{array}{c}1.63 \\
(0.87,3.06)\end{array}$ & $16(13.0)$ & $\begin{array}{c}0.27 \\
(0.15,0.46)\end{array}$ & $1.6(0.63 .4 .05)$ & $\begin{array}{c}2.94 \\
(1.67 .5 .18)\end{array}$ \\
\hline
\end{tabular}

Normal: individuals with normal pain sensitivity; reduced or enhanced: individuals with reduced or enhanced pain sensitivity $\mathrm{n}$, number of individuals; RR, risk ratio; RRR, relative risk ratio; CI, confidence interval; Ref, reference category

Note: excluded individuals where both reduced and enhanced sensitivity were reported

including missense/in-frame mutations within catalytic domain and TEY motif 